\title{
Critical reflection and abyssal trapping of near-inertial waves on a $\beta$-plane
}

\author{
Kraig B. Winters ${ }^{1} \uparrow$, Pascale Bouruet-Aubertot ${ }^{2}$ and Theo Gerkema ${ }^{3}$ \\ ${ }^{1}$ Scripps Institution of Oceanography, Mechanical and Aerospace Engineering. \\ University of California San Diego, La Jolla, CA 92093-0209. USA \\ ${ }^{2}$ Laboratoire d'océanographie et du climat: expérimentation et approches numériques, UPMC/CRNS, \\ BP 100. 4 place Jussieu. 75252 Paris CEDEX 05. France \\ ${ }^{3}$ Royal Netherlands Institute for Sea Research, NL-1790 AB Den Burg (Texel), The Netherlands
}

(Received 1 May 2010; revised 16 June 2011; accepted 30 June 2011)

We consider near-inertial waves continuously excited by a localized source and their subsequent radiation and evolution on a two-dimensional $\beta$-plane. Numerical simulations are used to quantify the wave propagation and the energy flux in a realistically stratified ocean basin. We focus on the dynamics near and poleward of the inertial latitude where the local value of the Coriolis parameter $f$ matches the forcing frequency $\sigma$, contrasting the behaviour of waves under the traditional approximation (TA), where only the component of the Earth's rotation aligned with gravity is retained in the dynamics, with that obtained under the non-traditional approach (non-TA) in which the horizontal component of rotation is retained. Under the TA, assuming inviscid linear wave propagation in the WKB limit, all energy radiated from the source eventually propagates toward the equator, with the initially poleward propagation being internally reflected at the inertial latitude. Under the non-TA however, these waves propagate sub-inertially beyond their inertial latitude, exhibiting multiple reflections between internal turning points that lie poleward of the inertial latitude and the bottom. The numerical experiments complement and extend existing theory by relaxing the linearity and WKB approximations, and by illustrating the time development of the steadily forced flow and the spatial patterns of energy flux and flux divergence. The flux divergence of the flow at both the forcing frequency and its first harmonic reveal the spatial patterns of nonlinear energy transfer and highlight the importance of nonlinearity in the vicinity of near-critical bottom reflection at the inertial latitude of the forced waves.

Key words: ocean processes, stratified flows, waves in rotating fluids

\section{Introduction}

We consider the behaviour of near-inertial oceanic internal gravity waves propagating meridionally in a depth-varying stratification toward their inertial latitude where the local value of the Coriolis parameter $f=2 \Omega \sin \phi$ matches the wave frequency $\sigma$. Here $\Omega$ is the frequency of the Earth's rotation and $\phi$ is latitude in spherical coordinates. Under the so-called traditional approximation (TA), the influence 
of the locally horizontal component of the Earth's rotation is neglected and the retained component of the rotation vector is everywhere aligned with the gravitational acceleration. On the $\beta$-plane, i.e. after making a tangent plane approximation to convert from spherical to Cartesian coordinates, the TA constrains gravitational and rotational effects to act in directions parallel or perpendicular to the coordinate directions and, assuming a rigid lid and a flat bottom for simplicity, to the domain boundaries.

One consequence of the TA is that throughout the domain, the allowable frequency range for propagating waves is $f<\sigma<N$ where $N$ is the local buoyancy frequency. This implies that poleward-propagating waves at a fixed frequency $\sigma<2 \Omega$ eventually reach their inertial latitude where $\sigma=f$ and approaching waves are internally reflected. Poleward of this latitude, the waves are sub-inertial and thus evanescent. Under the TA, all propagating waves are super-inertial and all wave energy flux is eventually directed equatorward (ignoring here the fate of waves that eventually cross the equator and propagate toward their inertial turning latitude in the opposite hemisphere; this situation is considered in e.g. Harlander \& Maas (2006) for equatorially trapped internal waves).

If one does not neglect the horizontal component of the rotation vector, an approach that we call non-traditional (non-TA) adopting the terminology of Gerkema et al. (2008), the rotation vector is no longer aligned with gravity, the coordinate directions nor the domain boundaries (except at the poles). This loss of symmetry has rather profound implications for the dynamics of near-inertial internal waves. In particular, propagating sub-inertial waves exist under the non-TA showing that the inertial latitude is not a turning latitude of internal reflection. In fact, energy propagates poleward of the inertial latitude until it arrives at its true turning latitude, defined by the transition of the underlying partial differential equation from hyperbolic to elliptic type. The location of the turning points, i.e. the position of the separatrix defining the transition, is shifted poleward from the inertial latitude by a distance that depends on the stratification and thus on depth. The shift can be of the order of hundreds of kilometres in the deep ocean and wave energy that crosses the inertial latitude can propagate sub-inertially, advancing poleward but multiply reflected from the internal turning locations and the bottom while being continually focused into an ever-thinning depth range above the bottom.

Adopting the more general non-TA therefore changes the description of oceanic near-inertial wave dynamics to order one. Instead of all poleward-propagating energy being internally reflected at the inertial latitude, a fraction of the energy is focused and trapped in the deep ocean. This was pointed out by Maas (2001), following studies on internal waves in rotating stratified spherical shells by Friedlander \& Siegmann (1982) and Dintrans, Rieutord \& Valdettaro (1999). The separatrix is curved in a thick spherical shell, but approaches a straight vertical (i.e. in the radial direction) in a uniformly stratified thin shell. With decreasing stratification $N$ with depth (as is natural in the ocean) the separatrix is curved even in a thin shell or on a $\beta$-plane.

The latter situation was examined in an earlier study (Gerkema \& Shrira 2005a), which was, however, based on three approximations or assumptions that will be relaxed in the present study. The first was the restriction to linear theory, which may be generally reasonable for small-amplitude waves, but can break down when wave beams reflect at boundaries or cross in the fluid interior. Moreover, in this problem one of the characteristics of near-inertial waves becomes exactly horizontal at the inertial latitude, so that, at a horizontal bottom, critical reflection will occur. Studies have shown that critical reflection leads, among other things, to the generation of higher 
harmonics (Dauxois \& Young 1999). Second, energy propagation was assumed to follow the characteristics without the occurrence of internal reflections; this amounts to making the WKB approximation, i.e. one assumes that the inhomogeneities of the medium (here due to vertically varying stratification $N$ and to the variation of the Coriolis parameter with latitude, the $\beta$-effect) occur at scales much larger than the wave itself, and therefore cause no internal reflections. This assumption becomes problematic near the inertial latitude, where horizontal wavelengths are long. Third, only the spatial problem was addressed, assuming sinusoidal waves varying like $\exp (\mathrm{i} \sigma t)$. This makes it impossible to say how long it takes for the patterns to form, especially in the region of abyssal trapping, where sub-inertial energy accumulates. The main purpose of the present paper is to demonstrate what happens if one abandons these three restrictions.

We explore here the fate of internal waves approaching the abyssal trapping zone through a series of idealized numerical experiments in which spatially localized monochromatic forcing is prescribed to excite waves that propagate meridionally on a $\beta$-plane. Energy is radiated from the forcing region both up- and downward and to the north (poleward) and south. We consider the solutions under linear and nonlinear dynamics and using the traditional and non-traditional treatments of the Coriolis effects. The simulations describe the approach toward steady state, allowing for the possibility of scale transformations and excitation of harmonics through nonlinear effects. Though our results apply to near-inertial waves generally, for specificity we consider the fate of semi-diurnal $\left(M_{2}\right)$ internal tidal beams generated within a few hundred kilometres of their inertial latitude. This particular context illustrates the importance of the horizontal component of rotation even at high latitudes in the deep ocean.

The remainder of the paper is organized as follows. First, we consider the characteristics obtained from the linearized equations of motion and show the direction of energy propagation for an idealized point source forcing. We describe the numerical methodology in $\$ 3$ and list the suite of experiments to be presented. Results for linear propagation under the traditional and non-traditional treatments of the Coriolis terms are contrasted in $\S 4$. In $\$ 5$ we discuss the companion experiments, in which the artificial restriction to linear dynamics is removed, and consider in particular the excitation and propagation of energy at the frequency of the first harmonic $2 \sigma$. We then focus on the trapping region, where waves at frequency $\sigma$ are sub-inertial and confined to a shrinking benthic region north of the $M_{2}$ inertial latitude. Discussion and conclusions follow in $§ 7$.

\section{Characteristics of the linearized equations}

To illustrate the spatial patterns of internal wave radiation under the combined effects of depth-varying stratification and latitudinally dependent rotation, we first examine the characteristic directions, i.e. directions in space along which linear waves transport energy. These are obtained from the linearized Boussinesq equations on a $\beta$-plane centred at latitude $\phi_{0}$ taking into account both components of the Earth's rotation vector. (The governing linear equations are obtained from (3.1) to (3.4) in $\$ 3$ after neglecting the nonlinear, forcing and dissipation terms.) We take coordinate directions $(x, y, z)$ pointing in the east, north and upward directions respectively with corresponding velocity components $u, v$ and $w$. The buoyancy $b=-\left(g / \rho_{0}\right) \rho^{\prime}$, where the prime indicates the space and time deviation from the ambient profile $\bar{\rho}(z)$ and $\rho_{0}$ is a constant reference density. In the tangent plane approximation, gravity acts only in the $z$ direction and the Coriolis parameter $f$ varies with meridional position 
according to $f=f_{0}+\beta y$ where $f_{0}=2 \Omega \sin \left(\phi_{0}\right), \beta=2 \Omega \cos \left(\phi_{0}\right) / R$ with $\Omega$ the Earth's rotation frequency and $R$ the Earth's radius. The horizontal component of rotation, i.e. the component oriented in the meridional direction, perpendicular to the gravitational acceleration, influences the dynamics through the terms proportional to $\tilde{f}=2 \Omega \cos \left(\phi_{0}\right)$. As in Grimshaw (1975), $\tilde{f}$ is taken as a constant, independent of $y$. Here and throughout we take $\phi_{0}=74.5^{\circ}$, the inertial latitude for the semi-diurnal tide, and so $f_{0}=M_{2}$ and $y=0$ at this latitude.

A second-order partial differential equation is obtained from the homogeneous, linearized version of (3.1)-(3.4) by seeking time-harmonic solutions proportional to $\mathrm{e}^{\mathrm{i} \sigma t}$ :

$$
A v_{y y}+2 B v_{y z}+C v_{z z}+\tilde{f} \beta v_{z}=0 .
$$

We have neglected zonal derivatives to focus on meridional propagation and eliminated variables in favour of the $y$ component of velocity $v(y, z)$. The coefficients of the equation are $A(z)=N^{2}(z)-\sigma^{2}+\tilde{f}^{2}, B(y)=\tilde{f f}$ and $C(y)=\left(f^{2}-\sigma^{2}\right)$. This is equation (2) in Gerkema \& Shrira (2005a). Note that the TA amounts to taking $\tilde{f}=0$ here, which eliminates $B$ and hence the mixed derivative term as well as the lower-order term proportional to $v_{z}$. In the more general non-TA, terms proportional to $\tilde{f}$ are retained.

No analytical solutions of (2.1) have been found for a vertically bounded domain. Exact solutions (Gerkema \& Shrira 2005b) have been obtained however for the $f$-plane where the $\beta$-effect is neglected (see also Kasahara 2003, 2004; Durran \& Bretherton 2004). For uniform $N$, Gerkema \& Shrira (2005b) obtained a simple but remarkable dispersion relation, according to which horizontal wavenumbers tend to infinity, and the group velocity to zero, as the wave frequency approaches its lower bound, which lies slightly below $f$. This is the sub-inertial short-wave limit. Translating this to the $\beta$-plane, the implication would be that wavenumbers go from zero at the inertial latitude to infinity at the turning latitude, no matter how small the distance between those latitudes may be. Whether this is a real physical effect, or rather an artefact of the WKB approach underlying this reasoning, will be examined in the present study.

Given a stratification profile $N(z)$, which we take here and throughout to be given by $N=N_{0} \mathrm{e}^{(z-H) / \gamma}$ with the surface value $N_{0}$ corresponding to 3 cycles h ${ }^{-1}$ and $\gamma=1300 \mathrm{~m}$, one can immediately display the characteristic directions

$$
\frac{\mathrm{d} z}{\mathrm{~d} y}(y, z)=\frac{B}{A} \pm \frac{\left(B^{2}-A C\right)^{1 / 2}}{A} .
$$

We will use the notation $\mu_{+}$and $\mu_{-}$to refer to families of characteristics with slopes given by the positive and negative branches of the square root respectively. For a given frequency $\sigma$, energy is constrained to propagate along characteristic directions regardless of spatial scale. Figure 1(a) shows the characteristics under the TA for meridional wave propagation at frequency $\sigma=M_{2}$ emanating from a hypothetical point source located at $1500 \mathrm{~m}$ depth at $y=-192 \mathrm{~km}$. The vertical dashed line at the $M_{2}$ inertial latitude $y=0$ indicates the transition between hyperbolic and elliptic regions for (2.1) under the TA, which is found generally by setting $B^{2}-A C=0$ in (2.2). Under the WKB approximation, arrows indicating the direction of wave radiation can be added to the characteristics as shown in the figure. In this limit, energy propagating northward is internally reflected at the inertial latitude in both the upper and lower ocean. After internal reflection, all energy propagates to the south as indicated by the filled arrows.

Figure 1(b) shows the corresponding characteristics under the non-TA in which the terms multiplied by $\tilde{f}$ are retained. The dashed line again shows the transition 

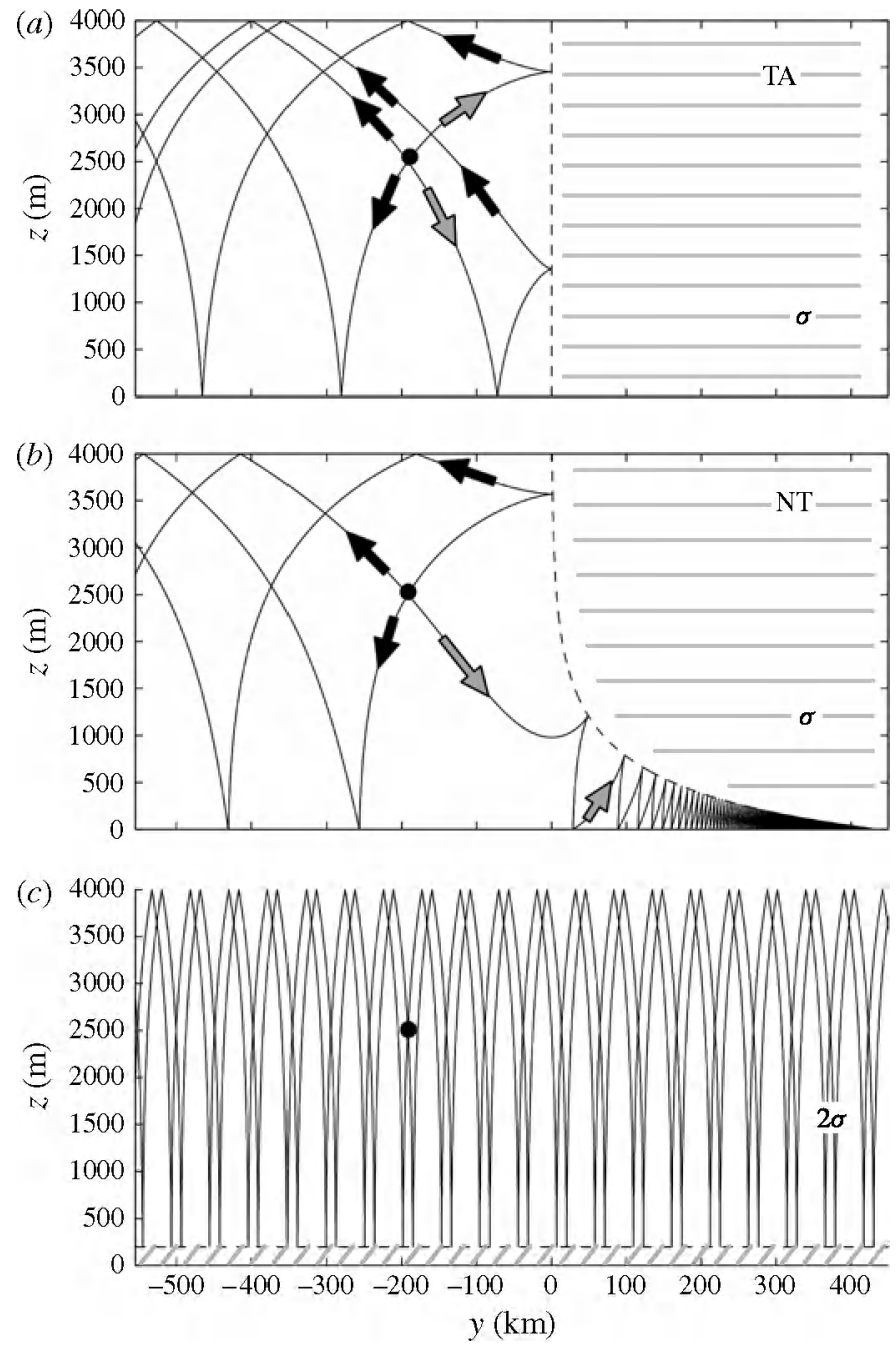

FIGURE 1. (a) Characteristics for linear WKB waves at frequency $\sigma=M_{2}$ on a $\beta$-plane under the traditional approximation (TA). The dashed line at $y=0$ is the separatrix between hyperbolic and elliptic regions (grey horizontal lines) which corresponds to the inertial latitude $y=0$ where $f=\sigma$. The forcing location is shown as a filled circle approximately $192 \mathrm{~km}$ south of the inertial latitude. $(b)$ Corresponding characteristics under the more general non-traditional approach. The turning locations, again shown as a dashed line, are shifted northward from the inertial latitude by an amount that increases with decreasing $N(z)$. (c) Characteristics at the first harmonic frequency $2 \sigma$ under the non-TA. Waves at $2 \sigma$ propagate along characteristics that are much steeper and are not limited in range by the latitudinal variation of $f$ over the range of latitudes shown.

between propagating and evanescent wave regions where (2.1) changes type. In this case however, owing to the retention of $B$, the transition is given by

$$
y(z)= \pm \frac{f_{0}}{\beta}\left(\sqrt{\frac{N^{2}-f_{0}^{2}+\tilde{f}^{2}}{N^{2}-f_{0}^{2}}}-1\right),
$$


indicating a northward shift from the inertial latitude that increases with decreasing $N$ in the deep ocean as shown in the figure. Energy that propagates initially northward and upward encounters a turning point in the upper ocean that is only slightly displaced from the inertial latitude and is reflected to the south. In contrast, energy that propagates initially northward and downward crosses the inertial latitude $y=0$ with a slope $\mathrm{d} z / \mathrm{d} y=0$ ( $C$ vanishes at the inertial latitude) where it becomes sub-inertial and approaches its true turning point while propagating upward. The downward reflected characteristic propagates to the south and there are two possibilities as discussed in Gerkema \& Shrira (2005a). If the characteristic intersects the bottom poleward of the inertial latitude, it is reflected upward and toward the pole. In this case, the characteristics eventually focus toward a point attractor as shown in the figure. This trapping is the $\beta$-plane analogue to the trapping in a rotating, stratified spherical shell found by Dintrans et al. (1999). The other possibility is that the ray intersects the bottom south of the inertial latitude. In this case (not shown here, see figure 4) the wave is super-inertial upon reflection and reflects upward but continues toward the south, thus escaping the point attractor. It is clear then, that the point attractor has a finite domain of attraction, within which all characteristics eventually focus and outside which energy is ultimately reflected back to the south. We do not explore this issue further here, focusing rather on a particular example in which energy radiates from a source located along a characteristic that lies within the domain of attraction.

Figure 1(c) shows the corresponding characteristics for waves at twice the forcing frequency emanating from the same source location though, as we will see, harmonics are not preferentially excited at that location. These characteristics are much steeper, appearing almost vertical in deep ocean for the aspect ratio of the figure. Waves at $2 \sigma$ are not limited in range by the latitudinal variation of $f$ within the domain shown. Their region of hyperbolicity is however limited by the decreasing value of $N$ with depth. The dashed line near the bottom indicates the region where the frequency $2 \sigma$ exceeds the maximum allowable frequency $\sigma_{\max }$.

When the linearity and WKB approximations are relaxed, the energy flux may not be as simple as indicated in figure 1. For example, waves at scales larger than those for which WKB applies may back reflect or scatter onto intersecting characteristics while nonlinearities can excite waves at other frequencies that propagate in different directions.

\section{Methodology}

We consider use following set of equations:

$$
\begin{gathered}
\frac{\mathrm{D} u}{\mathrm{D} t}-f v+\tilde{f} w=\mathscr{O}[u]+\mathscr{S}[u], \\
\frac{\mathrm{D} v}{\mathrm{D} t}+f u=-p_{y}+\mathscr{D}[v]+\psi(y, z, t), \\
\frac{\mathrm{D} w}{\mathrm{D} t}-b-\tilde{f} u=-p_{z}+\mathscr{D}[w]+\mathscr{S}[w], \\
v_{y}+w_{z}=0, \\
\frac{\mathrm{D} b}{\mathrm{D} t}+N^{2} w=\mathscr{D}[b]+\mathscr{S}[b] .
\end{gathered}
$$

Equations (3.1)-(3.5) are solved numerically in the two-dimensional $(\partial / \partial x \rightarrow 0)$ meridional plane for $-L_{y} / 2 \leqslant y \leqslant L_{y} / 2,0 \leqslant z \leqslant H$ for $0<t \leqslant T_{f}$ for the parameter values shown in table 1 . The pressure $p$ is scaled by the reference density $\rho_{0}$. 


$\begin{array}{lcccc} & \text { TA_lin } & \text { NT_lin } & \text { NT_nl } & \text { SI_nl } \\ L_{y}(\mathrm{~km}) & 1350 & 1350 & 1350 & 800 \\ H(\mathrm{~m}) & 4000 & 4000 & 4000 & 2000 \\ \mathrm{~d} y(\mathrm{~km}) & 1.3 & 1.3 & 1.3 & 1.56 \\ \mathrm{~d} z(\mathrm{~m}) & 3.9 & 3.9 & 3.9 & 3.9 \\ T_{f}(\mathrm{yr}) & 1 & 1 & 1 & 2 \\ \text { NT } & \text { No } & \text { Yes } & \text { Yes } & \text { Yes } \\ \text { NL } & \text { No } & \text { No } & \text { Yes } & \text { Yes } \\ (y, z)_{s r c}(\mathrm{~km}) & (-192,2.5) & (-192,2.5) & (-192,2.5) & (0,1.0) \\ a\left(\mathrm{~m}^{2}\right. & 50 & 50 & 50 & 20 \\ \nu\left(\mathrm{m}^{2} \mathrm{~s}^{-1}\right) & 10^{-5} & 10^{-5} & 10^{-5} & 10^{-6}\end{array}$

TABLE 1. Simulation parameters. Parameters that are held constant across all simulations are not listed. SI, subinertial.

The horizontal boundaries are taken to be free-slip walls with $u_{z}=v_{z}=w=b=0$. Even symmetry is assumed for all variables at $y= \pm L_{y} / 2$. While in principle such conditions are strongly reflective, we also impose Rayleigh damping in sponge layers adjacent to these boundaries to filter outward radiating waves before they reach the boundaries. This approach allows all variables to be expanded horizontally in cosine series and the use of accurate numerical methods. Unfortunately, despite the simplicity of the boundary conditions at $z=0$ and $H$, the non-TA precludes the use of a corresponding set of vertical expansions, i.e. $w$ and $b$ in sine and $u$ and $v$ in cosine series. The difficulty with this approach can be seen by looking at the polarity of (3.1) and noting that the term $\tilde{f} w$ would have odd symmetry while the remaining terms would be even in $z$. The underlying problem is that the use of sine expansions for $w$ implicitly assumes that all even derivatives of $w$ vanish, which is not the case using the non-TA. This also leads to the non-differentiability of Fourier series for non-traditional modes for $w$ on an $f$-plane as discussed in Gerkema \& Shrira (2005b). For this reason, we use sixth-order compact-differencing in the $z$ direction for which these symmetry issues are not a problem. These methods are global in the sense that the estimation of the value of the derivative at a single point $\left(y, z_{0}\right)$ requires function values at all discrete $z$ positions at the same $y$ location. The global nature of the method leads to desirable numerical properties and high accuracy (Lele 1992).

Equations (3.1)-(3.5) are simplified for some of the simulations. Linear simulations are those for which the material derivative $(\mathrm{D} / \mathrm{D} t)=(\partial / \partial t)+v(\partial / \partial y)+w(\partial / \partial z)$ is linearized to $\partial / \partial t$. For traditional (TA) simulations, the value of $\tilde{f}$ is set to zero while for non-traditional simulations $\tilde{f}=2 \Omega \cos \left(\phi_{0}\right) / R$. Included in the equations are an anisotropic dissipation operator $\mathscr{D}$, a forcing term $\psi$, and a Rayleigh damping or sponge term $\mathscr{S}$. The NL and NT labels for the parameters in table 1 indicate whether the nonlinear or non-traditional terms respectively are used in the various simulations.

The dissipation operator is defined as $\mathscr{D}=v\left(\partial^{2} / \partial z^{2}\right)+(-1)^{p-1} \tilde{v}\left(\partial^{2 p} / \partial y^{2 p}\right)$ for integer $p$ taken here to be 5. The higher-order operator is easily implemented in wavenumber space and takes advantage of the trigonometric expansions in $y$ to confine the effective dissipation to only the smallest scales represented on the grid. Modes with $y$ wavenumber $k$ decay under the action of the horizontal part of the operator like $\exp \left(-\tilde{v} k^{2 p} t\right)$. Given $p=5$, the value of $\tilde{v}$ is specified so that the mode at the Nyquist wavenumber decays by an amount $\mathrm{e}^{-1}$ in a time $T_{\text {diss }}=250 \mathrm{~s}$ which is about twice the time step used in most of the simulations. Using this value, motions with horizontal 
scale of $5 \mathrm{~km}$, which is $\sim 1 / 4$ the size of the horizontal forcing region and $\sim 4$ times the horizontal grid spacing, have a viscous decay time of $\sim 5 \mathrm{yr}$.

It is prohibitively difficult however to utilize such a scheme in the $z$ direction using compact differencing and so a conventional second-order operator is used instead. The characteristic time scale of decay for grid-scale motions is $\mathrm{d} z^{2} / \nu$ and for the full-depth simulations this value is equal to $\sim 17$ days. Similarly, motions at $125 \mathrm{~m}$ scale, $\sim 1 / 4$ the vertical extent of the forcing region, have decay time scales of the order of $50 \mathrm{yr}$. Based on the characteristic decay times, we see that the vertical diffusion operator is very weak. To a good approximation, both operators exert a negligible influence over the majority of the scales represented on the grid and the simulated dynamics can be considered representative of the nearly inviscid limit.

For all experiments except SInnl, forcing is applied to the $v$ equation only with the explicit form given by

$$
\psi(y, z, t)=\psi_{0} \mathrm{e}^{-\left(\left(y-y_{s r c}\right) / \sigma_{y}\right)^{2}} \mathrm{e}^{-\left(\left(z-z_{s r c}\right) / \sigma_{z}\right)^{2}} \cos (\sigma t),
$$

where $\sigma=M_{2}$ is the semi-diurnal forcing frequency, $\psi_{0}=a \sigma^{2},\left(y_{s r c}, z_{s r c}\right)$ is the centre of the forcing region and $\left(\sigma_{y}, \sigma_{z}\right)$ are characteristic spatial scales set to $10 \mathrm{~km}$ and $250 \mathrm{~m}$ respectively. Parameters with values not specified here vary between simulations and are listed in table 1.

Outward radiating waves are damped in sponge regions adjacent to the lateral boundaries via Rayleigh damping. The explicit form of the damping, e.g. applied to $w$, is

$$
\mathscr{S}[w]=-\frac{w}{T_{R}}\left(\mathrm{e}^{-((y+L y / 2) / \lambda)^{2}}+\mathrm{e}^{-((y-L y / 2) / \lambda)^{2}}\right)
$$

so that non-zero values are forced toward zero over a time scale $T_{R}$ which is set to $1000 \mathrm{~s}$. The spatial extent of these damping regions is approximately given by $\lambda$ which is set to $60 \mathrm{~km}$. These terms protect the interior of the domain from unwanted reflections at the symmetry boundaries. We do not apply Rayleigh damping in (3.2) to avoid explicitly forcing flow convergence and a corresponding compensating flow. The equations were solved in parallel using the flow-solve code discussed in Winters, MacKinnon \& Mills (2004) and used in MacKinnon \& Winters (2005), modified to permit the use of compact differencing in the $z$ direction.

\section{Overview of linear dynamics}

We focus first on a contrasting pair of linear simulations in which the effects of nonlinearities are artificially removed by neglecting the nonlinear terms in the governing equations. Waves are excited monochromatically at frequency $\sigma=M_{2}$ (but with the forcing imposed suddenly at $t=0$ ) from a location at $1500 \mathrm{~m}$ depth approximately $200 \mathrm{~km}$ south of the $M_{2}$ inertial latitude $y=0$. Both simulations are run for $1 \mathrm{yr}$ or 705 forcing periods $T_{M_{2}}=2 \pi / \sigma$. Figure 2 shows the meridional velocity $v$ after $50 T_{M_{2}}, 350 T_{M_{2}}$ and $705 T_{M_{2}}$ for the simulations TA_lin and NT_lin. In both cases, the purely horizontal orientation of the forcing projects onto both characteristic directions and we see velocity maxima (red) closely aligned with the characteristics derived from linear theory. After $50 T_{M_{2}}$, it is only apparent that the waves are tightly constrained to characteristics over a relatively small distance from the source. As time advances however, the velocity maxima become much more tightly focused on the characteristic directions throughout the domain. 

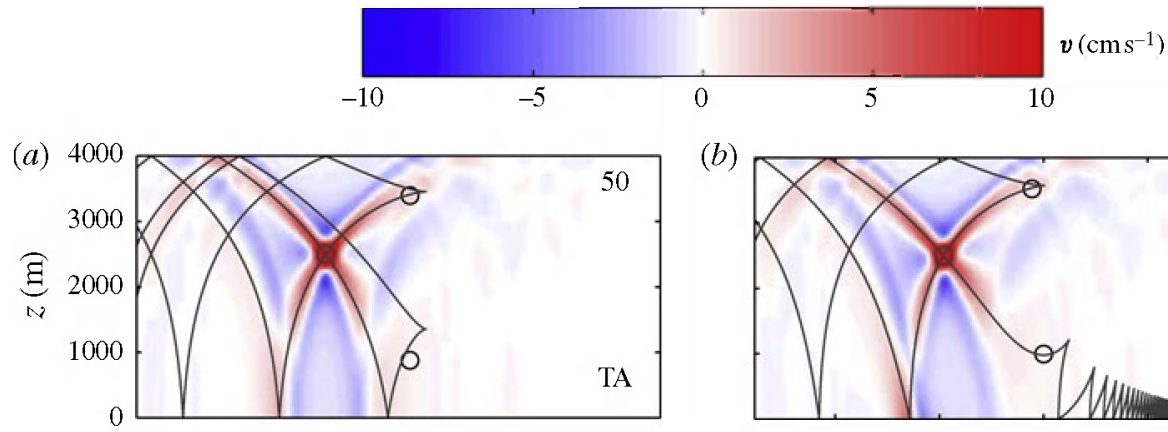

(b)
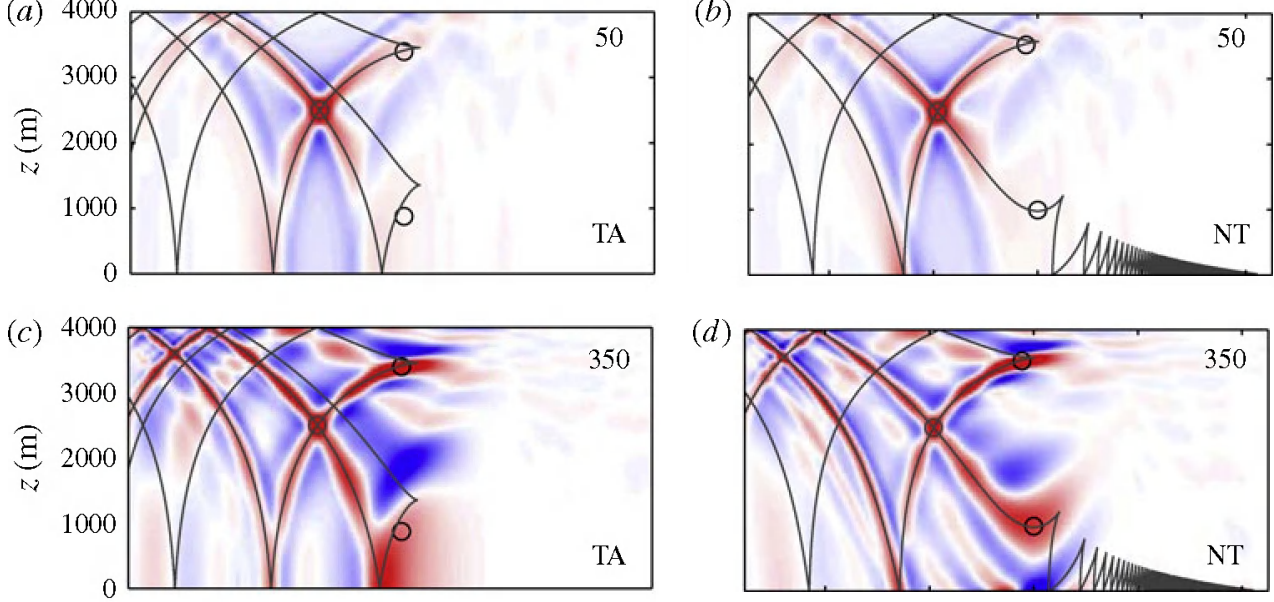

(d)

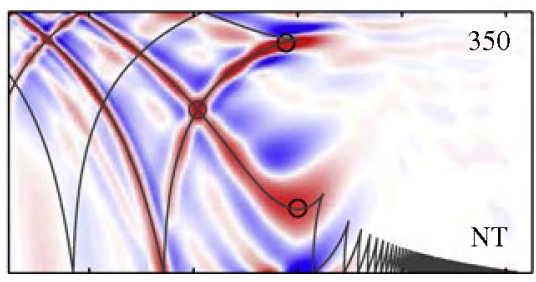

(e)
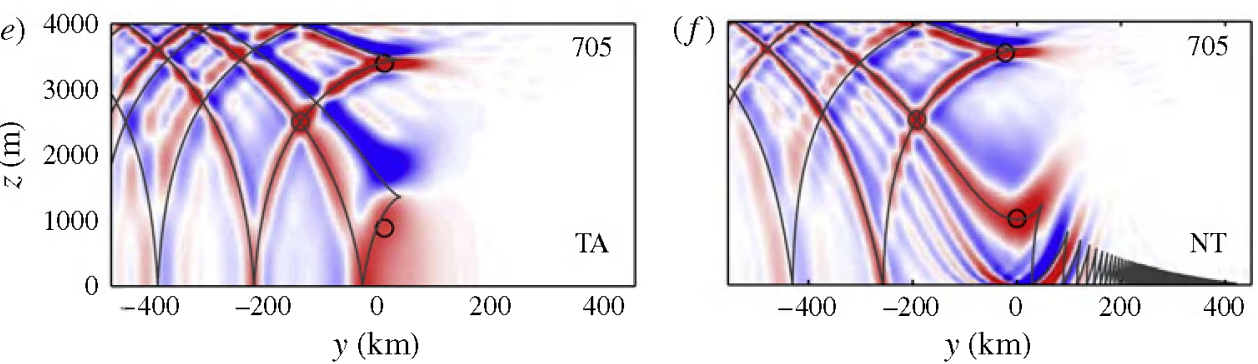

FIGURE 2. Snapshots of the meridional velocity $v$ in the $y, z$ plane after $(a, b) 50,(c, d) 350$ and $(e, f) 705$ forcing periods $T_{f}=2 \pi / \sigma$ for linear dynamics under the TA, $(a, c, e)$, and the NT approximation $(b, d, f)$. Also shown are the characteristics emanating from the centre of the source region for the forced frequency $\sigma=M_{2}$. The circles near $y=0$ are locations at which time series are extracted in figure 3.

The analyses of characteristics in $\S 2$ indicates that with the forcing applied here a turning point exists at a depth of $\sim 500 \mathrm{~m}$. In this region of relatively high stratification, the difference between TA and non-TA appears to be slight. Under the TA, this turning point is located at the $M_{2}$ inertial latitude $y=0$ while in the non-TA, it is shifted northward by an approximate distance $\delta=1.4 \mathrm{~km}$ which follows from (2.3) taking the value of $N$ at that depth.

While the early snapshots $\left(t=50 T_{M_{2}}\right)$ show no obvious evidence of energy being reflected at these locations, the later ones do. Velocity minima (blue) are formed along directions matching the reflected characteristics indicating a phase shift of $\pi$ upon reflection. The pattern of velocity maxima and minima sharpens significantly and becomes more tightly aligned with the predicted directions of energy reflection between $350 T_{M_{2}}$ and $705 T_{M_{2}}$. The linear behaviour is qualitatively similar for both cases near the upper-ocean turning point where $\delta$ is small compared to the characteristic forcing scale $\sigma_{y}$. Under the TA, WKB theory predicts an infinite horizontal wavelength as packets approach their inertial latitude $y=0$. The singularity 
however is an artefact of WKB theory. A less restrictive treatment, for example in the case of constant $N$, yields modal solutions in terms of Airy functions which can be interpreted in terms of incident, reflected and evanescent waves and no indication of a transformation to small scales. Without the TA, there is the additional complication that the WKB approach predicts infinitely short wavelengths a short distance $\delta$ north of the inertial latitude. Here however, even for constant $N$, no more general modal solutions have been found and so the possibility of very small scales cannot be formally dismissed. The numerical solutions however do not show any indication of energy transfer to small scales in this region and indeed, the solutions are very similar near the upper-ocean turning points whether or not the TA is employed. While confirmation is useful, this is perhaps not surprising given that $\delta \rightarrow 0$ as $N$ increases and one would strongly suspect that the two approaches yield the same result in this limit.

The behaviour near turning points in the deep ocean, where $N$ is much smaller and $\delta$ is much larger, however is significantly different between the two simulations. Under the TA, there is a simple turning point at $\sim z=1400 \mathrm{~m}$ where energy is reflected upward and to the south as indicated by the velocity minimum roughly centred along the predicted characteristic. The width of this reflected beam also decreases significantly between $350 T_{M_{2}}$ and $705 T_{M_{2}}$, particularly in the upper ocean well south of the inertial latitude, suggesting a continuous delay in arrival of small-scale waves at fixed locations. By $7057_{M_{2}}$, energy reflected from the deep turning point has reached the upper surface where it is reflected downward and southward.

In the NT simulation, energy is no longer internally reflected at the inertial latitude in the deep ocean. The $v$ maximum associated with downward and northward propagation is centred on the corresponding characteristic and crosses the inertial latitude with zero slope but finite group speed. The velocity maximum turns upward as the characteristics suggest. The $v$ signal decays above and to the north of the turning locations (see also figure 1) consistent with evanescence in the elliptic region of (2.1). Bands where $v$ alternates sign are evident within the focusing zone where characteristics reflect back and forth between turning points and the flat bottom. These bands are approximately aligned with the upward $\mu_{-}$characteristics in this region. Because $v$ is shown, rather than $w$ or energy density for example, it is not apparent in the figure that energy also propagates downward and southward along the predicted steep $\mu_{+}$characteristics. We will return to this point and consider the energy flux in the sub-inertial region in $\S 6$.

In the NT results, the flow below the $\mu_{-}$characteristic shown, including its extension upward and to the south of the forcing region, appears to be significantly different from that in the corresponding region under the TA. Under NT dynamics, distinct $v$ bands of alternating sign are evident that appear to lie along characteristics (not shown) from the $\mu_{-}$family in (2.2). The number of these bands approximately doubles between $350 T_{M_{2}}$ and $705 T_{M_{2}}$. The formation of these bands cannot be the result of nonlinear effects as the simulation is purely linear. They are strictly a consequence of the inclusion of the non-traditional Coriolis terms.

At the end of the simulations, the maximum values of $v$ are $\sim 15 \mathrm{~cm} \mathrm{~s}^{-1}$ in the forcing region, approximately twice this value in the positive $v$ (red) branch near the upper turning points. Near the inertial latitude in the deep ocean $(z \approx 1000 \mathrm{~km})$, values reach about 7 and $10 \mathrm{~cm} \mathrm{~s}^{-1}$ in the TA and NT simulations respectively. Time series of the $v$ velocity for the points near $y=0$ indicated with open circles in figure 2 are shown in figure 3. It is apparent that the approach to steady state, as indicated by the flow in the turning point regions is relatively slow. It might be argued that the NT 

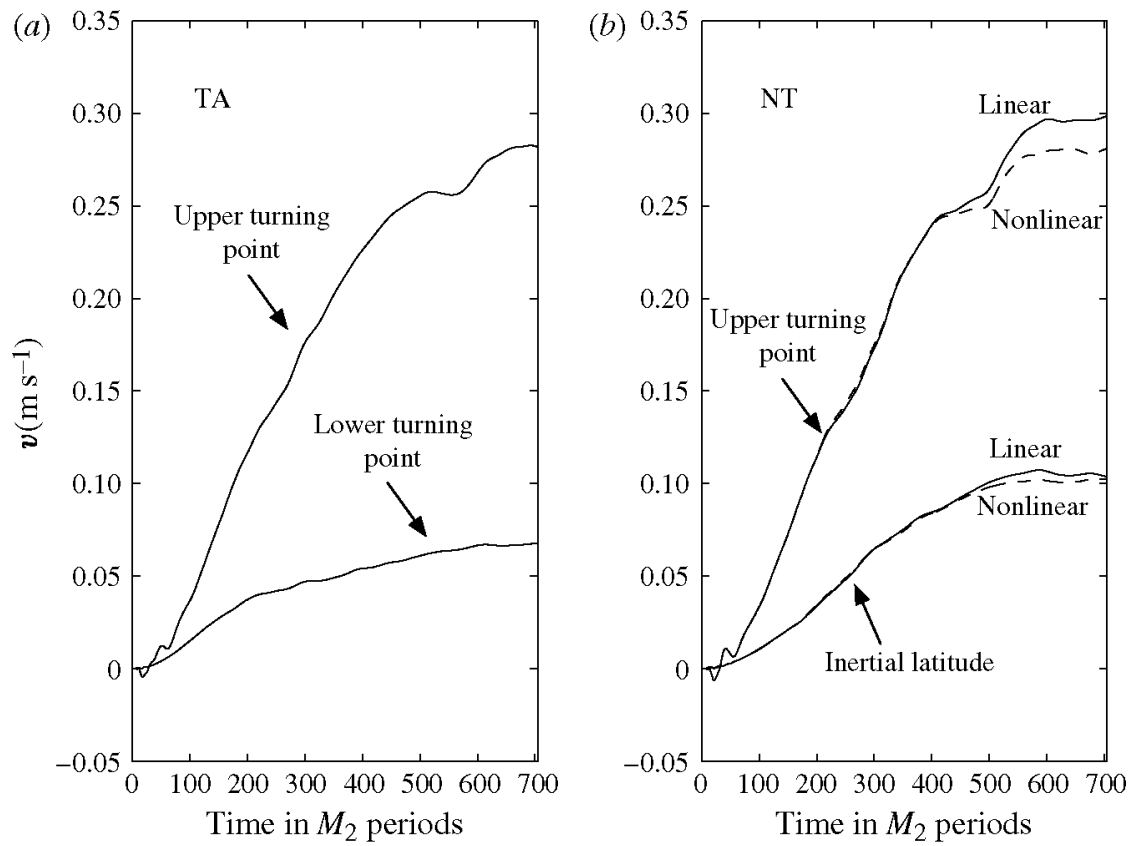

FIGURE 3. Time series of meridional velocity $v$, sampled once per forcing period $T_{f}$ at the locations indicated by the circles in figure 2 for $(a)$ the TA simulation and $(b)$ the NT simulation. For the NT simulation the time history for both linear and nonlinear experiments are shown.

simulations have attained an approximately steady state after 1 year; the development of the TA flow appears to be slightly slower. On the other hand, while the flow near the turning points may be still adjusting slowly due to the arrival of ever smallerscale waves, it is evident that significant energy has already been reflected from the upper turning points and has propagated well to the south of the excitation latitude. The extent to which the beams focus onto the predicted characteristics suggests that relatively small-scale waves have been able to travel the length of the reflected ray path in this time.

\subsection{Critical wave reflection}

We remarked in the previous section that the alternating bands seen in figure $2(d, f)$ resulted from the inclusion of the non-traditional Coriolis terms. We return to this point here. Figure 4 shows $\mu_{-}$characteristics slightly above and below the critical characteristics $\left(\mu_{-}\right)_{\text {upper }}$ and $\left(\mu_{-}\right)_{\text {lower }}$. (The critical characteristic $\left(\mu_{-}\right)_{\text {upper }}$ is sandwiched between the red and blue curves in the upper part of $(a)$ and is not shown for clarity.) Near $\left(\mu_{-}\right)_{\text {upper }}$, neighbouring trajectories just below (shown in red) reflect off the separatrix (dashed curve) and intersect the flat bottom just to the north of the inertial latitude $y=0$. (The critical characteristic $\left(\mu_{-}\right)_{\text {upper }}$ by definition intersects the separatrix at point A. Its reflection $\mu_{+}$then intersects the bottom at $y=0$.) These trajectories then reflect northward, eventually approaching the point attractor where the separatrix intersects the bottom (not shown). The inclusion of the non-traditional Coriolis terms alters the dispersion relation such that one gets a horizontal characteristic with finite group velocity at the inertial latitude. With a horizontal boundary, this implies critical reflection. While critical reflection occurs 


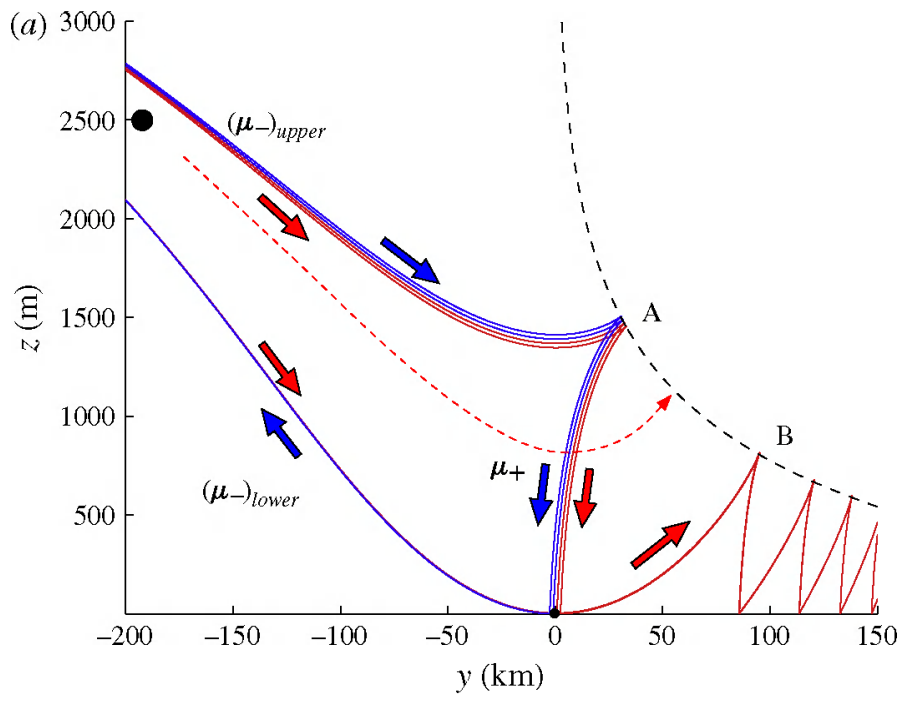

(b)

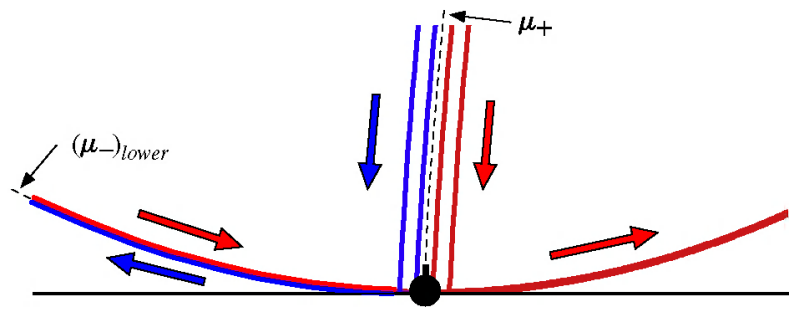

FIGURE 4. Characteristics adjacent to the critical characteristics that intersect the bottom at the inertial latitude $y=0$. (b) An enlargement of the lower part of $(a)$.

in many problems, one interesting feature of this problem is that the focused rays that result from near-critical reflection are then trapped within a waveguide, bounded by a physical boundary and an internal separatrix, that shrinks in spatial scale as it approaches a point attractor.

Also shown in red is a characteristic located just above $\left(\mu_{-}\right)_{\text {lower }}$ that passes the inertial latitude just above $z=0$. (By definition, the nearby critical or grazing characteristic $\left(\mu_{-}\right)_{\text {lower }}$ touches the bottom at $y=0$.) The point $(0,0)$ is therefore a point of critical reflection. It is well known (Eriksen 1985; Gilbert Garrett 1989; Ivey \& Nokes 1989; Slinn \& Riley 1998; Ivey, Winters \& De Silva 2000) that nonlinear interactions in the vicinity of critical reflection can lead to the generation of wave harmonics, energy intensification and turbulent mixing. Near-critical reflection acts to focus neighbouring characteristics, providing a simple mechanism for local intensification of energy density. A second interesting feature of this problem is that the critical reflection here has the property that it focuses energy from nonneighbouring rays from distinct locations in the water column, i.e. from near both $\left(\mu_{-}\right)_{\text {upper }}$ and $\left(\mu_{-}\right)_{\text {lower }}$. The merged ray bundle is then further concentrated as they propagate into a shrinking waveguide.

Figure $4(b)$ shows that the two red characteristics that originate near $\left(\mu_{-}\right)_{\text {upper }}$ are focused upon near-critical reflection and approximately merge with the distinct red characteristic from just above $\left(\mu_{-}\right)_{\text {lower }}$, with all three following essentially the same path into the sub-inertial region $y>0$ toward the point attractor. That energy from two 


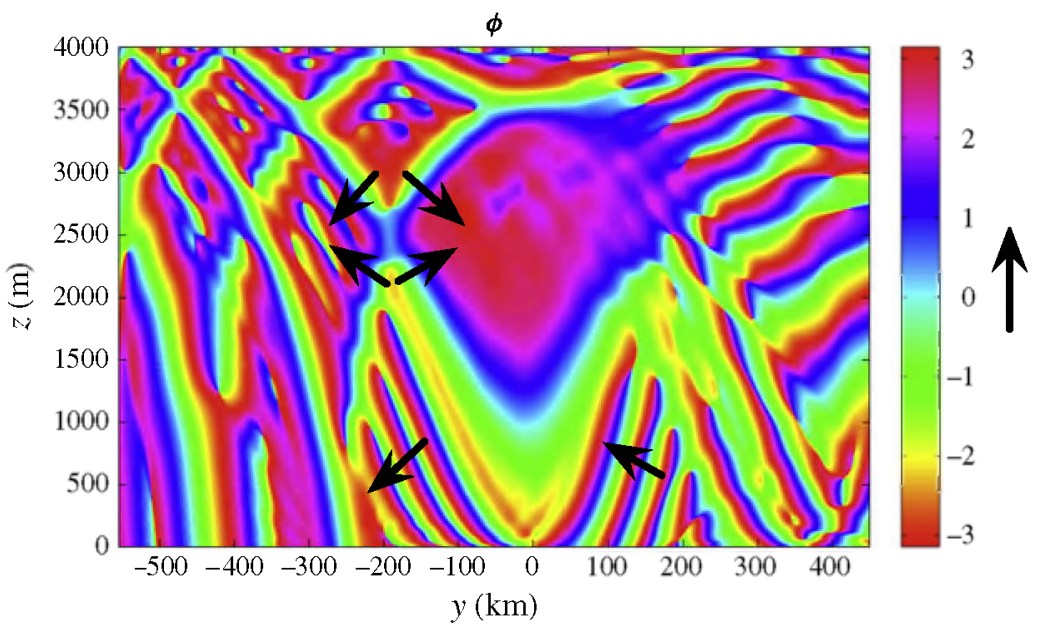

FIGURE 5. Phase diagram for $v$ corresponding to figure $2(f)$. Phase $\phi$ is shown in radians and the direction of phase propagation with time is indicated by the arrows.

distinct directions, propagating northward near $\left(\mu_{-}\right)_{\text {lower }}$ and southward along $\mu_{+}$, are focused upon reflection and directed into a vanishing waveguide makes this problem distinct from simple critical reflection of internal waves at a sloping boundary.

Similarly, characteristics just above $\left(\mu_{-}\right)_{\text {upper }}$ (shown in blue) reflect from the separatrix above the point labelled $\mathrm{A}$ and intersect the bottom just south of $y=0$ where they undergo a focusing near-critical reflection upward and to the south. Characteristics lying above the two blue ones shown will also reflect from the separatrix above A and from the bottom to the south of $y=0$ though with less focusing of neighbouring rays as the point of reflection moves south. The alternating bands of horizontal velocity visible in figure 2 (NT at $350 T_{f}$ and $750 T_{f}$ ) suggest that energy is indeed being transported along these characteristics after undergoing internal reflection at the separatrix.

For all points between $\left(\mu_{-}\right)_{\text {upper }}$ and $\left(\mu_{-}\right)_{\text {lower }}$, including for example the solid black circle indicating the centre of the forcing region in these simulations, the $\mu_{-}$characteristics all intersect the separatrix along the finite segment $\mathrm{AB}$ indicated in figure 4 , reflect downward, intersect $z=0$ to the north of $y=0$ and continue northward toward the point attractor.

The spatial structure of $v$ shown in figure $2(f)$ can be explained in terms of energy propagation and reflection along a bundle of rays that have turning points to either side of point A. Figure 5 shows the phase of $v$ computed over the last two periods of the run NT_lin. The four arrows in the upper part of the figure show the phase propagation with time for the beams that radiate energy away from the source region. For $y<0$, the forced waves are super-inertial and the directions of the phase and group velocities are vertically opposed. In the deep ocean, the phase propagation is down and to the south for $y<0$ implying energy propagation upward and to the south, as expected for waves reflected from the bottom. To the north of the critical point the forced waves are sub-inertial and, for $\mu_{-}$characteristics, the phase and group velocity directions are horizontally opposed (see table 1 in Gerkema \& Exarchou 2008). There the upward phase propagation is consistent with upward and northward energy propagation along a $\mu_{-}$characteristic into the sub-inertial trapping zone. 


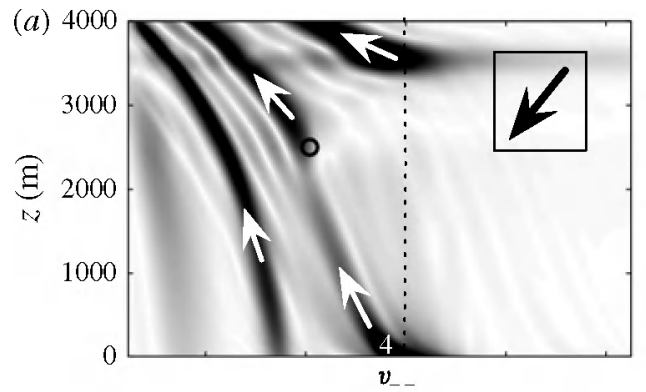

(b)

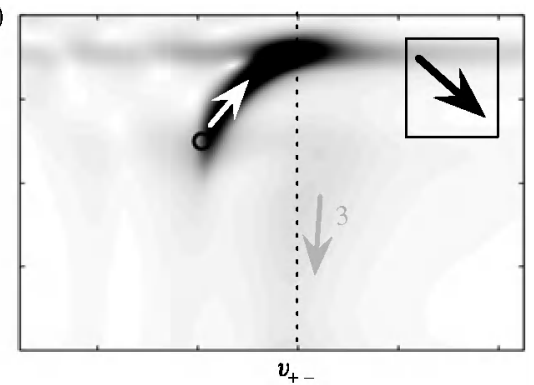

(c)

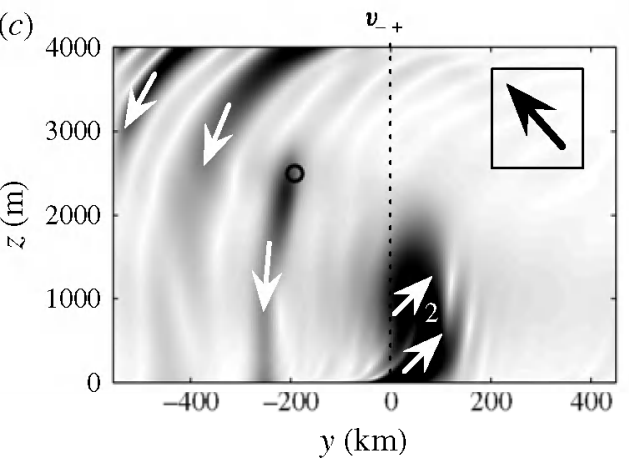

(d)

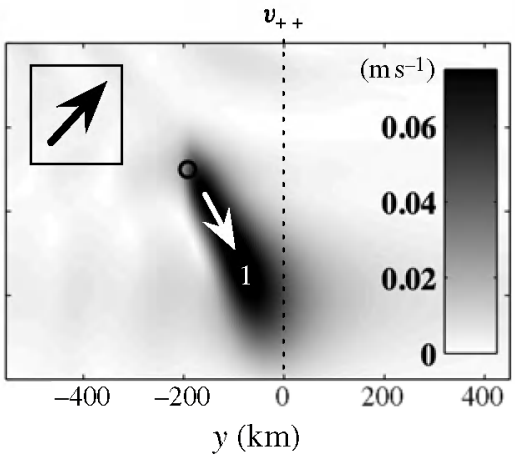

FIGURE 6 . The amplitude of $v$ corresponding to figure $2(f)$ decomposed by direction of phase propagation. Dark arrows show the direction of phase propagation, white (and grey) arrows show the direction of the group velocity. Subscripts refer to the sign of the $y$ and $z$ components of the phase velocity.

The amplitude and phase information can also be used to decompose $v$ into components with phase propagating in each of the four directions via a Hilbert transform (Mercier, Garnier \& Dauxois 2008). Figure 6 confirms the interpretation of the phase diagram. Energy radiates from the source downward and to the north (labelled 1, figure $6 d$ ). After crossing the inertial latitude it becomes sub-inertial and propagates upward toward the separatrix $(2$, figure $6 c$ ). It is then reflected downward and southward on steep $\mu_{+}$characteristics $(3$, figure $6 b$ ) though this branch does not appear very strongly in the decomposition for $v$. A portion of this energy intersects the bottom north of $y=0$, reflecting to the north $(2$, figure $6 c)$ while the remainder reflects south of $y=0$ and propagates upward and to the south $(4$, figure $6 a)$.

\subsection{Wave energy flux and its divergence}

We now consider the flux of energy transported by the waves. Let $\mathscr{E}$ be defined as

$$
\mathscr{E}=\frac{1}{2}\left(u^{2}+v^{2}+w^{2}+\frac{b^{2}}{N^{2}}\right) .
$$

Then, assuming that the vertical scale over which the waves vary is much smaller than that over which $N$ varies (otherwise additional terms, with derivatives of $N$, would come into play in the expression for energy density, e.g. Holliday \& Mcintyre 1981) the energy equation for (3.1)-(3.5) is

$$
\frac{\mathrm{D}}{\mathrm{D} t} \mathscr{E}=-\nabla \cdot \mathscr{F}+\Psi
$$


where $\mathscr{F}$ is the wave flux vector with horizontal and vertical components $\mathscr{F}_{h}=p v$ and $\mathscr{F}_{v}=p w$ and $\Psi=v \psi$ is the rate at which energy is supplied to the fluid via the forcing term in (3.2). For our purposes here we take the dissipative terms to be negligible, which is reasonable away from the sponge regions given the scales of the waves. We are interested in time-averaged quantities, which we indicate by angular brackets \langle\rangle . The time-averaging interval will be taken over the final 2 periods of the simulations when the flow is closest to a steady state. $\langle(\mathrm{D} / \mathrm{D} t) \mathscr{E}\rangle$ is thus the time-averaged rate of change of the energy density of a fluid parcel as it undergoes oscillatory motion. This time-averaged quantity is non-zero only if there is direct input or extraction via $\langle\Psi\rangle$ or when the divergence of the time-averaged wave flux $\langle\mathscr{F}\rangle \neq 0$. We note that $\langle\Psi\rangle>0$ within and negligible outside a small forcing region centred at $\left(y_{s r c}, z_{s r c}\right)$.

The horizontal and vertical components of the time-averaged wave energy flux $\langle\mathscr{F}\rangle$ in figure 7 show wave energy radiating outward from the forcing region in four directions. For the horizontal component, blue indicates southward propagation. Taking the time average over the last two $M_{2}$ periods in the simulation, integrating $\langle p v\rangle$ over the full depth at $y=100 \mathrm{~km}$ gives the net meridional flux in $\mathrm{W} \mathrm{m}^{-1}$. A steady balance between northward and reflected waves would give a net flux of zero. For the TA run the net flux is to the north, with a value slightly less than $10 \mathrm{~W} \mathrm{~m}^{-1}$. It is non-zero because the flow is still adjusting slowly near the turning points. In contrast, the NT run has a much larger northward flux of $\sim 65 \mathrm{~W} \mathrm{~m}^{-1}$ because much of the energy propagates into the trapping region. The two red bands north of $y=0$, one centred on the upward extent of the $\mu_{-}$ray emanating from the forcing zone and the second darker band reflected from the bottom near the point of critical reflection, indicate northward propagation of waves at locally sub-inertial frequency. The orientation of these bands is aligned with distinct $\mu_{-}$characteristic directions as discussed in $\S 4.1$.

The alternating red and blue bands in the trapping zone for the NT run figure $7(d)$ indicate propagation up and to the north and down and to the south between the separatrix and the bottom along $\mu_{-}$and $\mu+$ characteristics respectively. These patterns of horizontal and vertical flux confirm the interpretation of the characteristics in the sub-inertial region by showing that multiple reflections do occur in finite time and that, under linear dynamics at least, energy will eventually be transported northward toward the point attractor. Even after a year of steady forcing however, northward propagation to sub-inertial latitudes has not nearly traversed the approximately $400 \mathrm{~km}$ necessary to reach the point attractor.

Figure 7(e,f) shows the divergence of the flux $\langle\mathscr{F}\rangle$. In both the TA and NT cases, the flux divergence is strongly positive (red) in the forcing region $y \approx-200 \mathrm{~km}$, $z \approx 2500 \mathrm{~m}$ indicating that energy is radiated away from this region. Not shown is the corresponding rate of energy supply $\langle\Psi\rangle$ which has opposite sign and balances the outward radiation. Also apparent in figure $7(e, f)$ is a deep blue pattern near $y=-550 \mathrm{~km}$. These negative values indicate that more energy is radiated into these zones than is radiated away. Energy is not accumulated here however as the Rayleigh damping in the sponge region acts to dissipate the energy as it arrives, producing an approximately steady flow. (The left edge of the plot is $\sim 2 \lambda$ from the edge of the computational domain and is just within the sponge region.) The negative flux divergence near $y=-550 \mathrm{~km}$ therefore shows the energy flux that would escape the computational domain to the south were it not for the damping scheme imposed to avoid reflections. It is immediately apparent in comparing figures $7(e)$ and $7(f)$ that, for the same forcing, significantly more wave energy escapes equatorward under the 

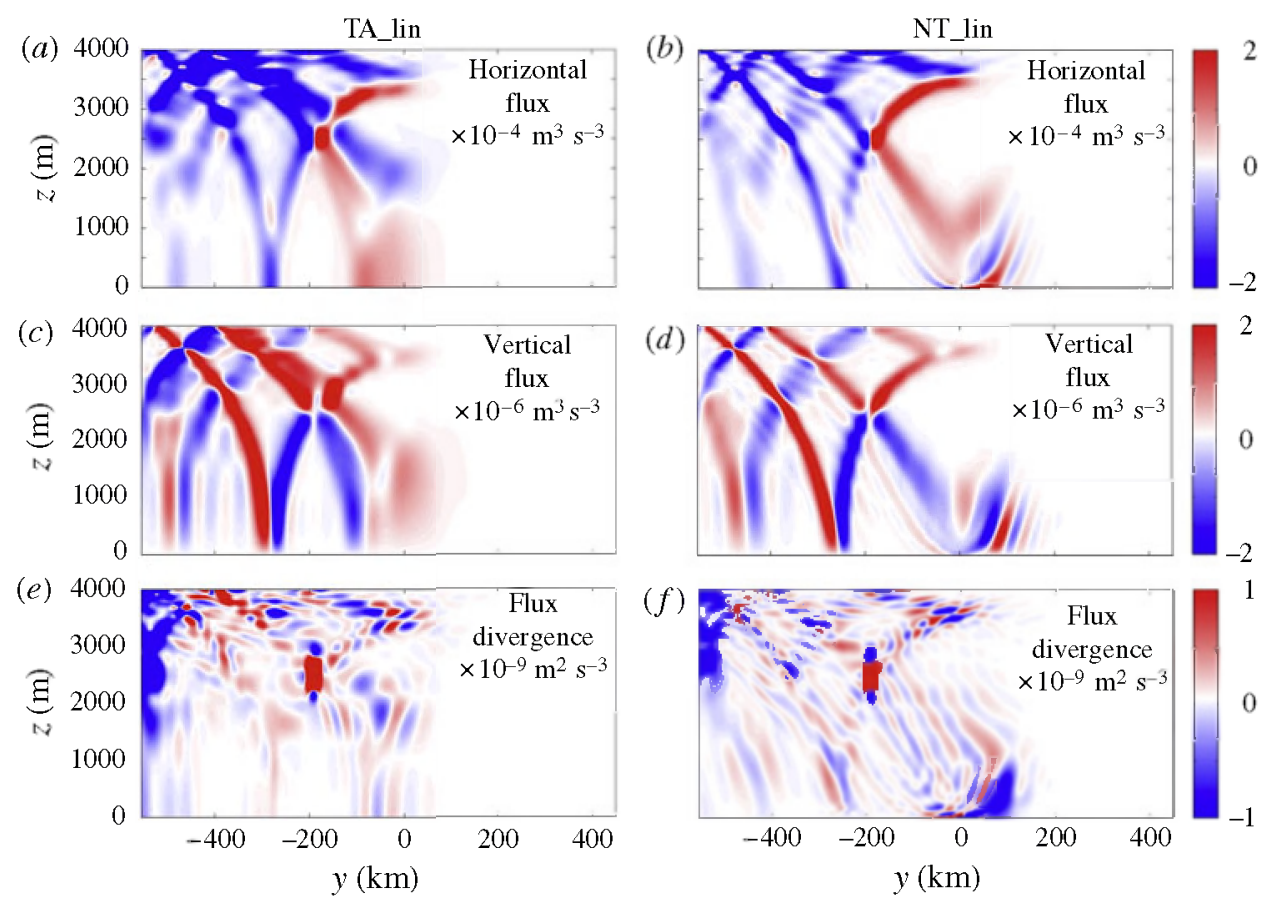

FIGURE 7. Time-averaged horizontal energy flux $\left\langle\mathscr{F}_{k}\right\rangle(a, b)$, vertical energy flux $\left\langle\mathscr{F}_{v}\right\rangle(c, d)$ and flux divergence $\nabla \cdot \overrightarrow{\mathscr{F}}(e, f)$ for the TA, $(a, c, e)$, and the NT, $(b, d, f)$ linear simulations. The sign convention is positive flux, i.e. northward or upward, shown in red, negative flux in blue.

TA than for the non-TA where a significant fraction of the energy is trapped at sub-inertial latitudes.

The strongly negative values for $y>0$ in the deep ocean in figure $7(f)$ also indicates that more energy is radiated into this zone than radiates away. In this region however, the Rayleigh damping term $\mathscr{S}$, which is spatially confined to the edges of the computational domain, is negligible. Furthermore, this energy is being transported by wave motions at spatial scales significantly larger than those affected by the dissipation operator $\mathscr{D}$. According to (4.2), energy must be accumulating in this zone and figure 8 , which indicates a rate of energy accumulation over the averaging interval of about $\Delta \mathscr{E} / \Delta t \approx 1.5 \times 10^{-9} \mathrm{~m}^{2} \mathrm{~s}^{-3}$, verifies this expectation. As we can see, the flow at sub-inertial latitudes is not yet steady after 1 year of continuous 'tidal' forcing.

\section{Overview of nonlinear dynamics}

In this section we discuss simulation NT_nl, the companion experiment to NT_lin in which the nonlinear advection terms are restored to the equations of motion. All other experimental parameters, including the forcing intensity, are unchanged. In general, nonlinearities induce motions at different frequencies and one might expect the primary frequencies excited to be the subharmonic $\sigma / 2$ and the first harmonic $2 \sigma$. In this problem however, the wave source is located relatively near the inertial latitude for the forced frequency. The sub-harmonic $M_{2} / 2$ has an inertial latitude of $28.8^{\circ}$ and waves of this frequency are evanescent throughout the computational domain. We therefore focus our attention here on the component of the flow at frequency $2 \sigma=M_{4}$. 


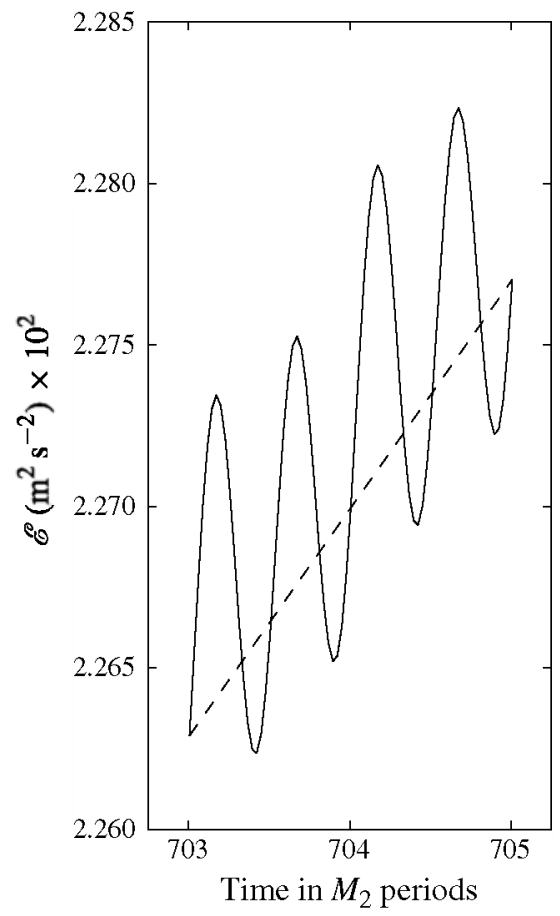

FIGURE 8. Energy density $\mathscr{E}(t)$ during the last two forcing periods for the NT_lin at the point $y=68.6 \mathrm{~km}, z=82 \mathrm{~m}$ which lies in the sub-inertial zone of energy accumulation of figure $7(f)$.

To decompose the flow into frequency components, we use the ansatz

$$
u_{\omega}(y, z, t)=a(y, z) \cos (\omega t)+b(y, z) \sin (\omega t)
$$

and compute the coefficients $a, b$ by multiplying by the time-harmonic components and integrating over an integer number of periods $2 \pi / \omega$. Coefficients were calculated for both the fundamental frequency $\sigma$ and the first harmonic $2 \sigma$ for the velocity components $(u, v, w)$ as well as for $b$ and $p$.

Figure 9 shows the $2 \sigma$ component of the meridional velocity $v$ computed over the final two forcing periods in the year-long simulation. The spatial pattern of this component is quite different from the purely linear results in figure 2 . While the overall pattern is consistent with the much steeper characteristics directions for waves at this frequency, a detailed correspondence between parts $(e)$ and $(f)$ of figures 1 and 9 is not evident. In particular, it is not obvious in the figure where the harmonic component of the flow is excited.

This component of the flow appears to be somewhat intensified in the upper ocean at $\sim y=-500 \mathrm{~km}$, just above the location where upward and downward southbound characteristics at frequency $\sigma$ (emanating from the prescribed source region) cross. More significant intensification is evident in the deep ocean just to the north of the inertial latitude $y=0$. Here it is immediately apparent that the intensified flow at frequency $2 \sigma$ occurs in alternating bands that appear to be aligned with $\mu_{-}$characteristics associated with the fundamental frequency $\sigma$. These bands are most intense in the deepest few hundred metres where waves at frequency $2 \sigma$ are evanescent owing to the weak near-bottom stratification. They also extend upward into 


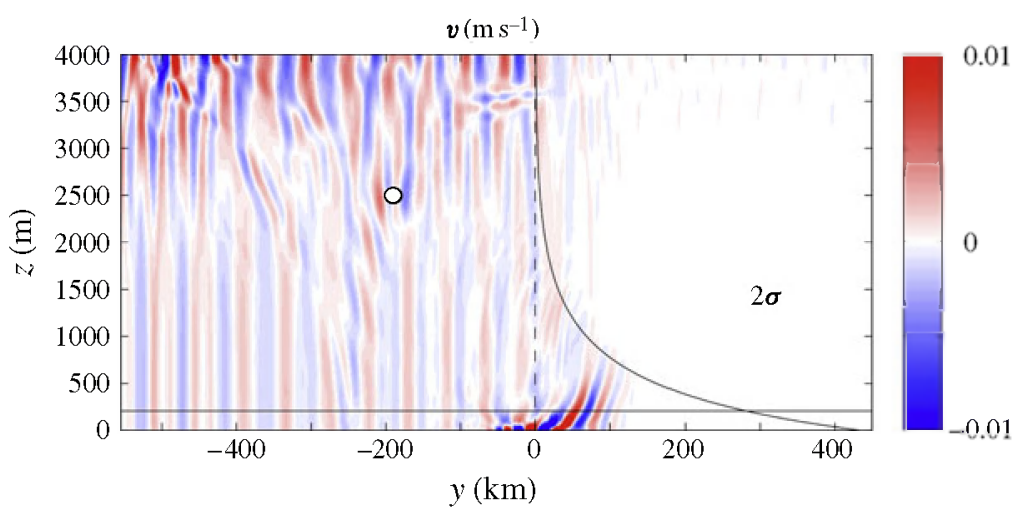

FIGURE 9. The $2 \sigma$ component of the meridional velocity $v$ at $t=705 T_{f}$. Also shown are curves separating propagating from evanescent wave regions according to linear theory for waves of frequency $\sigma$ (curved line) and frequency $2 \sigma$ (horizontal line near bottom).

the region where $2 \sigma$ waves can, in principle, propagate freely but decay rapidly above the separatrix marking the turning points for the directly forced waves at frequency $\sigma$, suggesting direct coupling to the $\sigma$ waves themselves. Similarly, while the $2 \sigma$ component of $v$ extends past the $\sigma=M_{2}$ inertial latitude in the upper ocean, the magnitude of this signal is significantly reduced. Finally, the predominant pattern consistent with steep $2 \sigma$ characteristic slopes is disrupted in places by phase lines with much shallower slope corresponding to the local characteristic directions at the forced frequency. This is particularly apparent just south of $y=0$ in the upper ocean where the forced waves are internally reflected as previously discussed.

Figure 10 shows the wave energy flux carried by the $2 \sigma$ component of the flow with the sign convention that negative flux values indicate propagation to the south and down in parts $(a)$ and $(b)$ respectively. While the vertical flux shows roughly equal flux in the positive and negative directions, the horizontal flux is predominantly southward and distributed rather diffusely in space though concentrated in the upper ocean. Northward flux, in contrast, is much more localized to distinct, relatively small patches that appear to align with the $\sigma$ characteristics radiating from the source location. The horizontal flux in the deep sub-inertial zone appears almost exclusively northward. The strongest vertical fluxes are found in the region $-550 \mathrm{~km}<y<-400 \mathrm{~km}$ beneath the locations where $\sigma$ characteristics reflect from the surface and intersect. Figure 10(c) shows the flux divergence. Positive values (red) indicate generation regions for $2 \sigma$ motions. The strongest sources exhibit phase patterns that correlate most strongly with reflections from the upper surface, the turning point in the upper ocean, and interior intersections of $\sigma$ characteristics. A significant source is also evident very near the point of critical reflection $(0,0)$. The flow in this region will be considered at higher resolution in the subsequent section.

\section{The trapping zone}

We now consider the flow in the deep ocean at sub-inertial latitudes in more detail. In simulation SI_nl, we force the flow from a region centred at the inertial latitude $1000 \mathrm{~m}$ above the bottom, a location approximately corresponding to the centre of the somewhat diffuse northward beam visible in figure $2(f)$. While in the previous simulations forcing was applied only to the $v$ component of velocity, both $v$ 

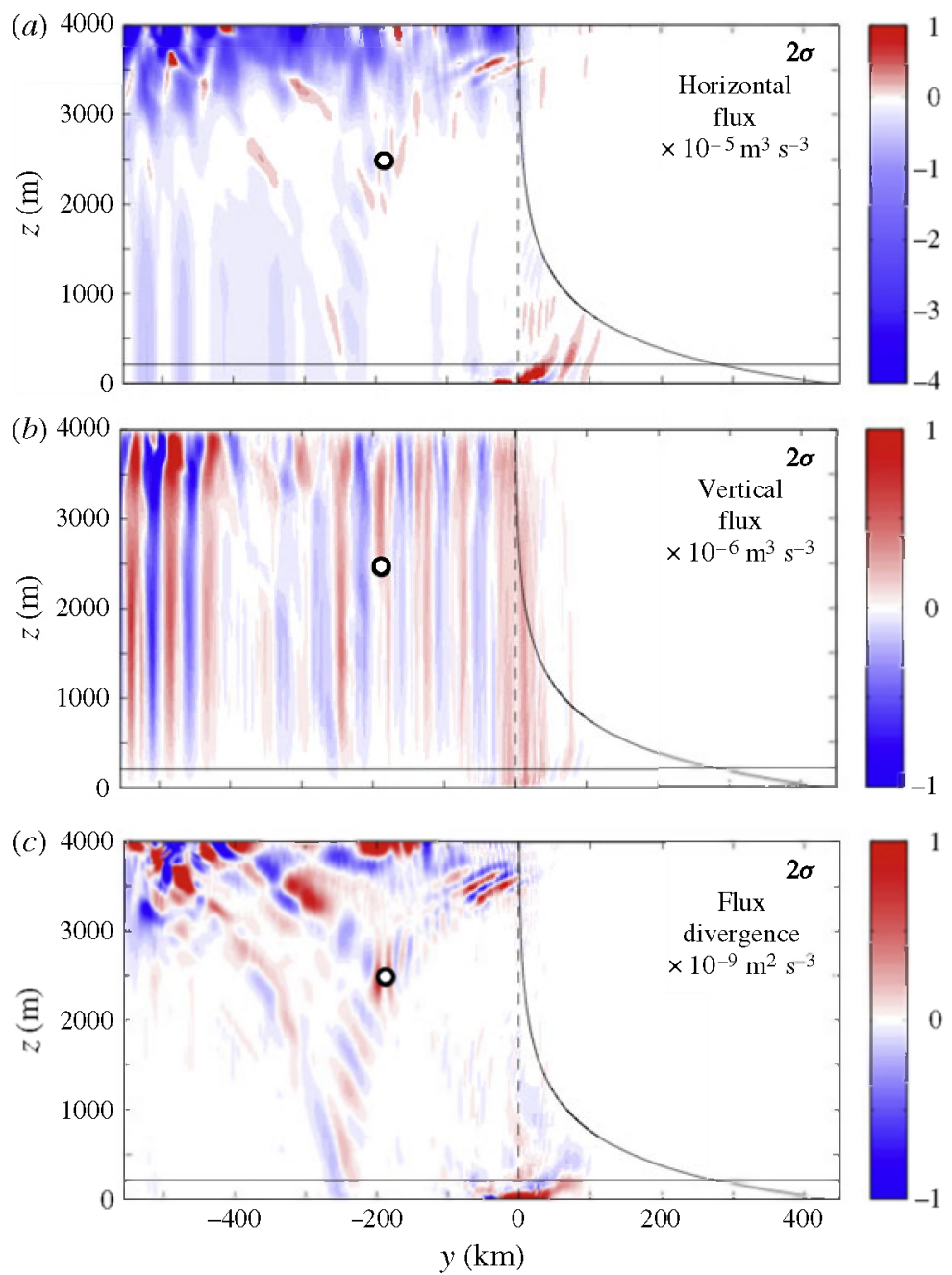

FIgURE 10. $(a, b)$ Energy flux and (c) flux divergence as in figure 7 but for the component of the flow at frequency $2 \sigma$ in simulation NT_nl.

and $w$ components were forced here such that the particle paths were aligned exactly perpendicular to the steep $\mu_{+}$characteristic at the centre of the source region. Energy was therefore radiated both to the north and to the south concentrated on the $\mu_{-}$ characteristic passing through the centre of the forcing region. Of course because the forcing region has finite size, we also expect energy to propagate along neighbouring characteristics, all of which have zero slope at the inertial latitude $y=0$.

The vertical extent of the domain is limited here to $2000 \mathrm{~m}$ and, in principle, energy encountering the artificial lid would reflect back into the domain. North of $y=0$, internal reflections at the separatrix prevent upward propagating energy from reaching the upper rigid lid and contamination of the interior flow by surface reflections is therefore minimal. Energy that propagates to the south encounters a sponge layer with strong Rayleigh damping within $\sim 100 \mathrm{~km}$. In this way, the experiment highlights the fate of energy that is focused toward the sub-inertial point attractor. 

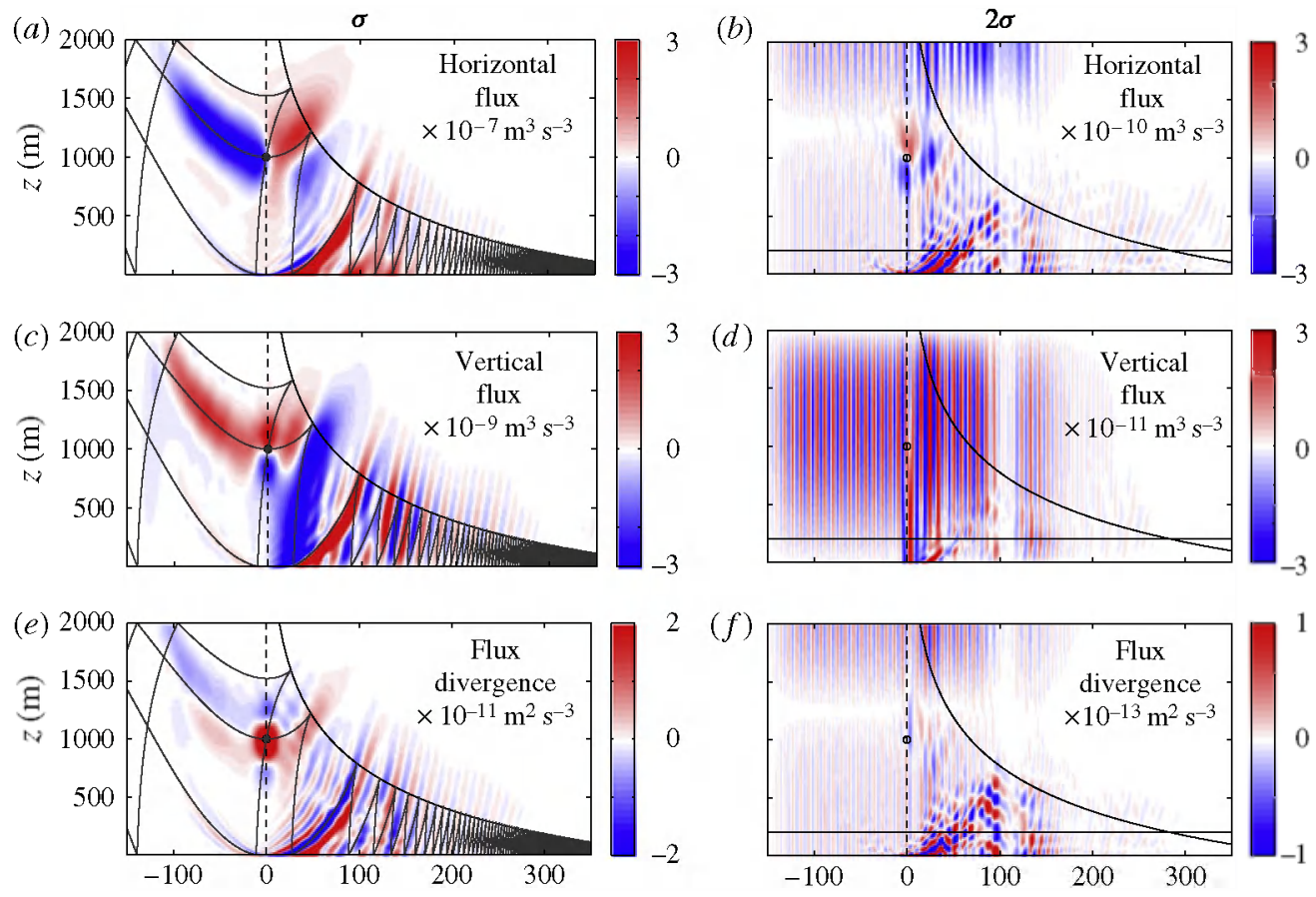

FIGURE 11. Energy flux and flux divergence as in figures 7 and 10 but for simulation SI_nl.

Figure 11 shows the components of the wave flux $\langle\mathscr{F}\rangle$ and the corresponding divergence computed after decomposing the flow into the directly forced $(\sigma)$ and harmonic $(2 \sigma)$ components. The averaging interval was taken as the last four periods $2 \pi / \sigma$ in the two-year integration. At frequency $\sigma$, figure 11(c) now clearly shows energy flux downward and southward along the steep $\mu_{+}$characteristic following the first internal reflection. Positive flux, both upward and to the north occurs along bands parallel to $\mu_{-}$characteristics, in particular along the distinguished characteristic that is tangent to the bottom, i.e. critically reflected at $(0,0)$. These bands decay above and to the north of the separatrix where internal reflection is observed. While multiple alternating bands are visible at sub-inertial latitudes after 2 years, it is still apparent that the bulk of the energy has not yet reached the attractor point. It is also apparent that simply following the central characteristic from the source region does not completely reproduce the spacing of the observed bands. This presumably results from forcing over finite scales and therefore over a set of nearby characteristics. Interestingly, in comparison with the larger-scale simulation NT_lin, the energy flux along upward and southward $\mu_{-}$characteristics emanating from near the point of critical reflection is very weak. Our interpretation is that the finite-sized forcing region in this simulation is somewhat more compact than the rather diffuse beam that reaches the inertial latitude in NT lin. In that simulation, more energy reaches the separatrix above the point $A$ indicated in figure 4 and therefore propagates along $\mu_{+}$ characteristics that intersect the bottom south of $y=0$, thus reflecting upward and to the south. The comparatively focused beam excited in this run is concentrated along characteristics that intersect the separatrix below the point $\mathrm{A}$, intersect the bottom north of $y=0$, reflect up and to the north and are therefore trapped.

Figure 11(e) shows the flux divergence at the forced frequency $\sigma$. The localized red patch in the forcing region again indicates wave radiation away from the source. 
There are also two strong bands of opposite sign that are closely aligned with a nearly critical $\mu_{-}$characteristic. Negative values indicate a convergence of energy; more energy propagates into these regions than leaves at frequency $\sigma$. This is what would be expected if energy were being transferred from the $\sigma$ component to the $2 \sigma$ component. Figure $11(b, d, f)$ shows the flux and divergence at the $2 \sigma$ frequency and we do indeed see that the strongest horizontal flux and divergence correspond with the regions of strong flux divergence at the fundamental frequency. The situation is not completely clear however owing to the alternating signs in the signals at both frequencies. This could indicate that there is an exchange of energy between the frequency components, i.e. from $\sigma \rightarrow 2 \sigma$ but also from $2 \sigma \rightarrow \sigma$ perhaps via parametric subharmonic instability (PSI) (McComas \& Bretherton 1977; BouruetAubertot et al. 2001; Gerkema, Staquet \& Bouruet-Aubertot 2006). It is clear however that the region near the point of critical reflection is a zone of strong coupling between the near-inertial waves and their harmonic.

\section{Discussion}

On the $\beta$-plane, retaining the horizontal component of rotation de-aligns the rotation and gravity vectors and alters the dispersion relation for inertio-gravity waves. While one might expect that the horizontal component of rotation would play a significant role at the equator, where its value is largest and the vertical component vanishes, it can also play a significant role in the dynamics of near-inertial internal waves, even at relatively high latitudes. Linear theory in a flat two-dimensional basin with realistic stratification shows that near-inertial waves are able to propagate poleward of their inertial latitude and can be focused and trapped in the deep weakly stratified ocean. The specific configuration considered, with localized mid-depth body forcing, can be thought of as an idealization of internal wave beams generated near the crest of an isolated, steep topographic feature by oscillating tidal currents at the semi-diurnal frequency. Energy radiated toward the poleward abyss, $\sim 25 \%$ of the energy radiated from the forcing region, is focused and trapped in the deep ocean.

In this idealized configuration, there are two special locations: the point of critical bottom reflection, which occurs at the inertial latitude for the forced wave; and the point attractor where the separatrix intersects the bottom. These points are of particular interest because there are clear mechanisms for intensification of wave energy densities (focusing near-critical reflection and waveguide shrinkage), wave nonlinearities are intrinsically important in their vicinity and they are therefore likely candidate locations for wave-driven deep ocean mixing. The simulations presented give qualitative confirmation of expected energy transmission paths, verifying the existence of internal reflections without making a WKB scale-separation approximation. They also confirm that the infinite/zero wavelength predictions at the inertial latitude and separatrix respectively are artifacts of WKB theory, that ray focusing and energy intensification does occur near points of critical reflection and that nonlinearities occur preferentially in the neighbourhood of these points. While suggestive, the simulations are not however sufficiently resolved to make direct statements about dissipation and mixing rates.

It should be noted that these results were obtained under steady background conditions for continuous forcing in the absence of other flows. Even so, the largerdomain simulations were only approximately steady near the inertial latitude after one year with the waves propagating only about half the distance from the inertial latitude to the point attractor in that time. Even for the smaller-domain run that focused 

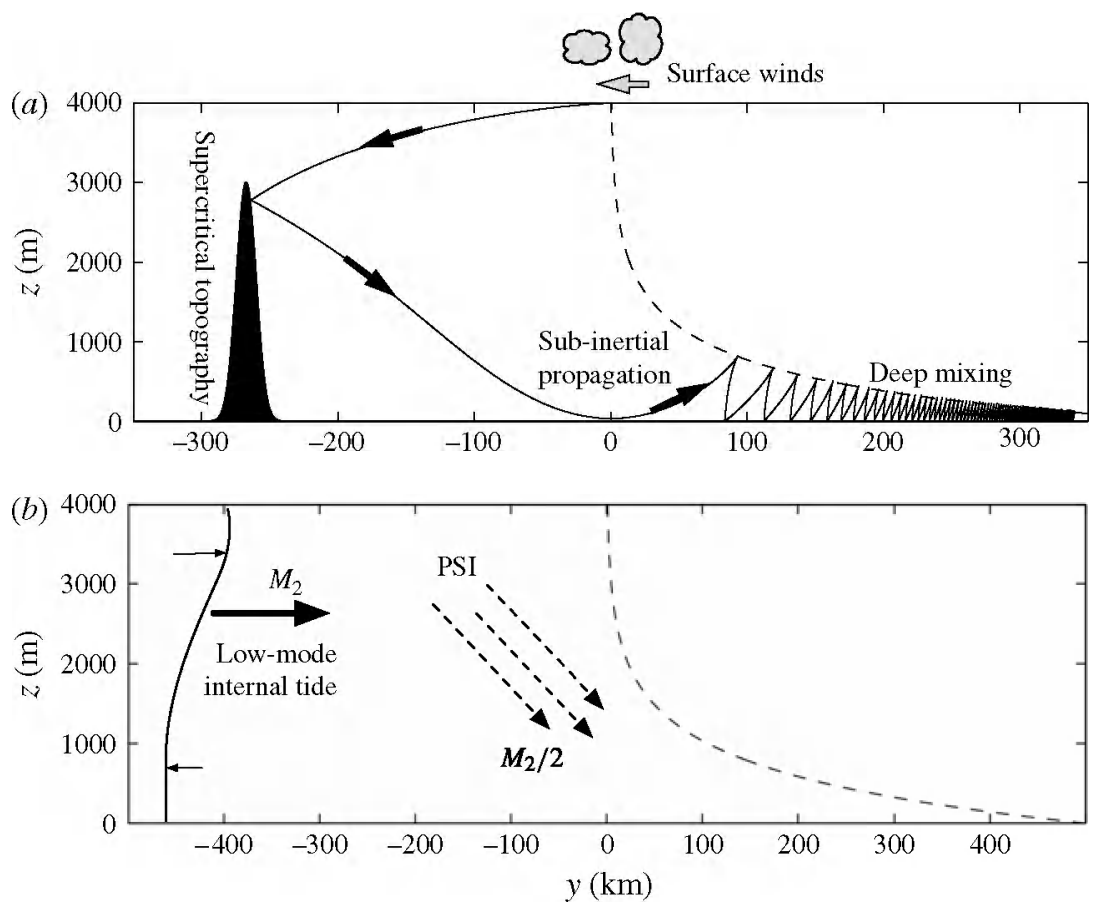

FIGURE 12. (a) Schematic showing the trapping of wind-generated inertial waves in the deep ocean poleward of the source. $(b)$ Schematic showing the trapping of near-inertial waves generated by PSI of the internal tide in the upper ocean. Here $y=0$ corresponds to the inertial latitude $28.8^{\circ}$ for waves at frequency $M_{2} / 2$.

directly on the trapping region, wave energy has not arrived at the attractor point after two years. While tidal forcing is continuous in time, the validity of the assumption of steady background conditions is perhaps suspect though if it were to be reasonable anywhere, one might expect it to be so in the abyss.

The behaviour of waves near their inertial latitudes in the deep ocean is not limited however to internal tides. Winds and storms at the surface force nearly horizontal surface currents in the surface mixed layer that then radiate into the deep ocean as near-inertial internal waves (D'Asaro et al. 1995). Because in the upper ocean (below the mixed layer) $N$ is typically large, the inertial latitude and the true turning point for inertial-frequency waves are very close, these waves are generated essentially at their turning latitudes and cannot propagate poleward, thus propagating equatorward into the ocean interior as shown in figure $12(a)$. We have already seen that the abyssal trapping region has a finite zone of attraction. Shown in the figure is a characteristic for a surface-generated inertial wave propagating into the interior along a $\mu_{+}$characteristic. The characteristic intersects a localized topographic feature with supercritical slope, i.e. a slope such that the forward reflecting characteristic would penetrate the topography and is thus disallowed. Upon reflection, energy is radiated along a $\mu_{-}$characteristic which, in this example, lies just above the critical ray that grazes the point of critical reflection $(0,0)$ and enters into the abyssal trapping region. This problem is analogous to the tidally forced problem except that the time scale of the wind forcing is both intermittent and of relatively short duration, perhaps of the order of days. Nonetheless, the combination of non-traditional effects and supercritical 


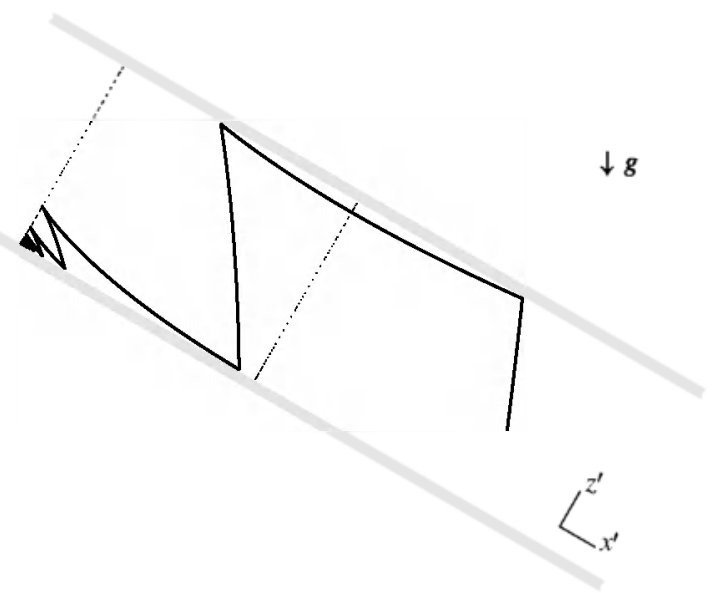

FIGURE 13. A stratified, tilted system, in which the isopycnals of the background stratification (two examples of which are indicated by the dotted lines) are perpendicular to the boundaries (in grey). Stratification decreases upslope, i.e. in the negative $x^{\prime}$ direction. Characteristics were calculated using an equation analogous to (2.2). There are two positions in the along-slope direction that are of particular importance. One is where one of the characteristics becomes parallel to the slope (level indicated by dotted line on the right). The other is the point beyond which waves cannot propagate anymore because stratification has become too weak: the transition from the hyperbolic to the elliptic regime (level indicated by the dotted line on the left); here waves get trapped near the bottom.

topography provides a potential mechanism for directly transferring energy from the wind to the poleward deep ocean. Unlike the tidally driven problem, where the point of critical reflection occurs at a single latitude, critical reflection of wind-driven inertial waves can potentially occur at any forcing latitude provided of course that the local topography is favourable.

Figure $12(b)$ illustrates another way in which wave energy can radiate into the sub-inertial trapping region. A propagating low-mode internal tide at frequency $M_{2}$ is subject to parametric subharmonic instability (PSI) as it propagates poleward on a $\beta$ plane. This nonlinear interaction transfers energy from the internal tide to smaller-scale waves at the subharmonic frequency $M_{2} / 2$ at latitudes equatorward of the $M_{2} / 2$ inertial latitude $28.8^{\circ}$ (e.g. MacKinnon \& Winters 2005). For realistic ocean stratification, PSI of the internal tide is an upper ocean phenomenon that radiates subharmonic, near-inertial waves to the deep ocean. This problem has recently been simulated under the TA by Hazewinkel \& Winters (2011), who show that these waves are internally reflected at the inertial latitude for all depths when non-traditional effects are ignored. The fate of these waves when non-traditional effects are included has not yet been addressed though (2.3) indicates that sub-inertial propagation might extend several hundred kilometres poleward of $28.8^{\circ}$ in the deep ocean.

In this paper we have focussed on a particular example of near inertial waves on the $\beta$-plane, in which non-traditional effects provide the means for symmetry breaking. Analogous flows can be constructed without variable rotation. A specific example is provided in the Appendix.

K.B.W. was supported by the National Science Foundation (grant number OCE 04-25283) and the Office of Naval Research (grant number N00014-05-1-0573). This 
work was also supported by CNRS (Centre National de la Recherche Scientifique) (LEFE/IDAO) and ANR (Agence Nationale pour la Recherche) (OPTIMISM project), as well as IDRIS (Institut du Développement et des ressources en Informatique, contract no 0111396) and the Netherlands Organization for Scientific Research (NWO) Grant 047.017.2006.003 for Dutch Russian Research Cooperation. We also thank V. Shrira, L. Maas and H. van Haren for interesting and useful discussions.

\section{Appendix. An analogue of the non-traditional $\beta$-plane}

Veronis (1970) pointed out an analogy between the effects of rotation and stratification, noting an exact mathematical correspondence between certain nonrotating, stratified flows and non-stratified, rotating flows.

The effect of non-traditional terms also has an analogue in non-rotating flows. As pointed out by Maas $(2001,2005)$, the key effect of the non-traditional terms on the $f$-plane lies in their symmetry breaking: the rotation axis is no longer aligned with gravity. A similar symmetry breaking can be created in non-rotating fluids by introducing a sloping boundary. Two types of attractors are here to be distinguished. One takes the form of a closed trajectory; for this, a supercritical slope is needed. The other is a point attractor, which occurs over a subcritical slope (e.g. internal waves running into the apex of a wedge). Similarly, the two types of attractors may occur on the non-traditional $f$-plane, for instance in a closed rectangular basin aligned to gravity; the closed-trajectory type of attractor would occur for super-inertial waves, the point attractor for sub-inertial waves (in one of the corners of the basin).

In the present paper, we have the additional complication of the variation of $f$ with latitude, i.e. the $\beta$-effect. This case also has an analogy, which is illuminating because it translates the less familiar non-traditional effects into more common terms. The analogy can be developed formally, yielding an equation of the same type as (2.1), but here we give only a qualitative description.

We consider a basin with parallel bottom and surface, both flat but tilted with respect to the vertical (figure 13). This tilting, by itself, has already created a symmetry breaking analogous to that on the non-traditional $f$-plane. We furthermore add a density stratification with sloping isopycnals such that they are normal to the tilted bottom (i.e. $\rho\left(x^{\prime}\right)$ ); this contrived situation requires rotation (about the gravitational axis) to keep the sloping isopycnals in place with a geostrophic current. We confine our attention here to high-frequency internal waves that are not significantly affected by rotation; in other words, the only role of rotation here is to have the isopycnals oriented normal to the tilted boundary.

At this point, internal wave characteristics would be straight. We alter this by adding a final ingredient, taking non-uniform stratification with the density gradient $\partial \rho\left(x^{\prime}\right) / \partial x^{\prime}$ decreasing linearly in the upslope direction. As a result, waves cannot propagate indefinitely leftward, but at some point encounter a location $x^{\prime}$ where the density gradient becomes too weak to support waves of a given frequency (left dotted line in figure 13). As shown in the figure, waves then become trapped in the corner in an entirely similar way as on the non-traditional $\beta$-plane, with the separatrix between hyperbolic and elliptic behaviour playing the same role in both cases.

Prior to reaching this point, there is an $x^{\prime}$ location (indicated by the right dotted line) at which one of the characteristics becomes parallel to the slope. To the right and left of this line, the boundary slope is super- and sub-critical respectively. This location is the analogue of the inertial latitude on the non-traditional $\beta$-plane. 
In summary, in this example the occurrence of wave trapping towards a point attractor requires two ingredients: symmetry breaking due to the tilt of the boundaries with respect to gravity, and along-slope variation of the density gradient, creating a separatrix between hyperbolic and elliptic regimes. Together, they allow a transition from super- to sub-criticality in the upslope direction, and focusing toward a point attractor.

In the preceding sections, the analogues of these two ingredients were the nontraditional Coriolis terms, creating a symmetry breaking due to the tilt of the rotation axis with respect to gravity, and the $\beta$-effect, which results in a separatrix. Together, they allow a transition from super- to sub-inertial waves in the poleward direction, and a point attractor at the sepatrix in the sub-inertial domain.

\section{REFERENCES}

Bouruet-Aubertot, P., Koudella, C., Staquet, C. \& Winters, K. B. 2001 Particle dispersion and mixing induced by breaking internal gravity waves. Dyn. Atmos. Oceans 33, 95-134.

D’Asaro, E. A., Eriksen, C. C., Levine, M. A., Niller, P., Paulson, C. A. \& van Meurs, P. 1995 Upper ocean inertial currents forced by a strong storm. Part I: data and comparisons with linear theory. J. Phys. Oceanogr. 25, 2909-2936.

Dauxois, T. \& Young, W. R. 1999 Near critical reflection of internal waves. J. Fluid Mech. 390, 271-295.

Dintrans, B., Rieutord, M. \& VAldettaro, L. 1999 Gravito-inertial waves in a rotating stratified sphere or spherical shell. J. Fluid Mech. 398, 271-297.

DURran, D. \& Bretherton, C. 2004 Comments on 'The roles of the horizontal component of the Earth's angular velocity in nonhydrostatic linear models'. J. Atmos. Sci. 61, 1982-1986.

ERIKSEN, C. C. 1985 Implications of ocean bottom reflection for internal wave spectra and mixing. J. Phys. Oceanogr. 15, 1145-1156.

FRIEDLANDER, S. \& SIEGMANN, W. L. 1982 Internal waves in a rotating stratified fluid in an arbitrary gravitational field. Geophys. Astrophys. Fluid Dyn. 19, 267-291.

GERKEMA, T. \& EXARCHOU, E. 2008 Internal-wave properties in weakly stratified layers. J. Mar. Res. 66, 617-644.

GERKEMA, T. \& SHRIRA, V. I. $2005 a$ Near-inertial waves on the 'nontraditional' $\beta$-plane. $J$ Geophys. Res. 110, C01003.

GERKEMA, T. \& SHRIRA, V. I. $2005 b$ Near-inertial waves in the ocean: beyond the traditional approximation'. J. Fluid Mech. 529, 195-219.

Gerkema, T., Staquet, C. \& Bouruet-Aubertot, P. 2006 Decay of semi-diurnal internal-tide beams due to subharmonic resonance. Geophys. Res. Lett. 33, L08604.

Gerkema, T., Zimmerman, J. T. F., MaAs, L. R. M. \& van Haren, H. 2008 Geophysical and astrophysical fluid dynamics beyond the traditional approximation. Rev. Geophys. 46, RG2004.

Gilbert, D. \& GARRETT, C. 1989 Implications for ocean mixing of internal wave scattering off irregular topography. J. Phys. Oceanogr. 19, 1716-1729.

GRIMSHAW, R. H. J. 1975 A note on the $\beta$-plane approximation. Tellus 27, 351-357.

HARLANDER, U. \& MAAS, L. R. M. 2006 Characteristics and energy rays of equatorially trapped, zonally symmetric internal waves. Meteorol. Z. 15 (4), 439-450.

HAZEWINKEL, J. \& WiNTERS, K. B. 2011 PSI of the internal tide on a $\beta$-plane: flux divergence and near-inertial wave propagation. J. Phys Oceanogr. (accepted).

Holliday, D. \& MCINTYRE, M. E. 1981 On potential energy density in an incompressible, stratified fluid. J. Fluid Mech. 107, 221-225.

IVEY, G. N. \& NoKES, R. I. 1989 Vertical mixing due to the breaking of critical internal waves on sloping boundaries. J. Fluid Mech. 204, 479-500.

IVEY, G. N., Winters, K. B. \& DE SilvA, I. P. D. 2000 Turbulent mixing in a sloping benthic boundary layer energised by internal waves. J. Fluid Mech. 418, 59-76. 
KASAHARA, A. 2003 The roles of the horizontal component of the Earth's angular velocity in nonhydrostatic linear models. J. Atmos. Sci. 60, 1085-1095.

Kasahara, A. 2004 Reply to Comments on 'The roles of the horizontal component of the Earth's angular velocity in nonhydrostatic linear models'. J. Atmos. Sci. 61, 1987-1991.

LeLe, S. K. 1992 Compact finite difference schemes with spectral-like resolution. J. Comput. Phys. $103,16-42$.

MAAs, L. R. M. 2001 Wave focusing and ensuing mean flow due to symmetry breaking in rotating fluids. J. Fluid Mech. 437, 13-28.

MAAS, L. R. M. 2005 Wave attractors: linear yet nonlinear. Intl J. Bifurcations Chaos 15 (9), $2757-2782$.

Mackinnon, J. A. \& Winters, K. B. 2005 Subtropical catastrophe: significant loss of low-mode tidal energy at $28.9^{\circ}$. Geophys. Res. Lett. 32, L15605.

MCCOMAS, C. H. \& BRETheRTon, F. P. 1977 Resonant interaction of oceanic internal waves: the dynamic balance of internal waves. J. Geophys. Res. 82, 1397-1412.

Mercier, M. J., Garnier, N. B. \& DauXois, T. 2008 Reflection and diffraction of internal waves analyzed with the Hilbert transform. Phys. Fluids 20, 086601.

SLINN, D. N. \& RiLeY, J. J. 1998 Turbulent dynamics of a critically reflecting internal gravity wave. Theor. Comput. Fluid Dyn. 11, 281-303.

Winters, K. B., MacKinnon, J. A. \& Mills, B. 2004 A spectral model for process studies of rotating, density-stratified flows. J. Atmos. Ocean. Technol. 21, 69-94.

Veronis, G. 1970 The analogy between rotating and stratified fluids. Annu. Rev. Fluid Mech. 2, $37-66$. 